\title{
EVOLUTION OF A LONG-TRACK VIOLENT TORNADO WITHIN A SIMULATED SUPERCELL
}

Leigh Orf, Robert Wilhelmson, Bruce Lee, Catherine Finley, and Adam Houston

Utilizing state-of-the-art visualization and analysis software, we explore the evolution of a violent tornado within a simulated supercell thunderstorm and describe associated computational challenges.

U nderstanding the processes involved in the genesis, maintenance, and decay of tornadoes within supercells remains an active research topic because of the loss of life and extreme damage they cause. Recent field campaigns, such as Verification of the Origins of Rotation in Tornadoes Experiment 2 (VORTEX2) (Wurman et al. 2012), have provided insight into these

AFFILIATIONS: ORF-Cooperative Institute for Meteorological Satellite Studies, University of Wisconsin-Madison, Madison, Wisconsin; WILHELMSON-University of Illinois at Urbana-Champaign, Urbana, Illinois; LeE-High Impact Weather Research and Consulting, LLC, Deep River, Minnesota; FINLEY-Saint Louis University, St. Louis, Missouri; Houston-University of Nebraska-Lincoln, Lincoln, Nebraska

CORRESPONDING AUTHOR E-MAIL: Leigh Orf, leigh.orf @ssec.wisc.edu

The abstract for this article can be found in this issue, following the table of contents.

DOI:10.1175/BAMS-D-I5-00073.I

A supplement to this article is available online (10.1175/BAMS-D-15-00073.2)

In final form 29 April 2016

(C)2017 American Meteorological Society processes, but forecasting tornadogenesis within an already-formed supercell remains a formidable challenge.

Seminal numerical simulations of supercell thunderstorms conducted in the 1970s and 1980s (e.g., Klemp and Wilhelmson 1978a,b; Schlesinger 1980; Rotunno and Klemp 1982; Weisman and Klemp 1982, 1984; Rotunno and Klemp 1985) were the basis from which scientific theories of supercell formation, strength, and maintenance emerged (Wilhelmson and Wicker 2001). Contemporary model-based studies have built on this knowledge base through introduction of increasingly sophisticated and realistic treatments of storm dynamics/physics.

Highly idealized numerical models run at extremely high resolution, but over small domains, have been used to explore the dynamics of analytically forced, fully resolved tornadoes absent of an explicitly resolved parent storm (e.g., Lewellen et al. 1997, 2000; Fiedler 1994; Rotunno 2013). In these simulations, only the tornado and its immediate environment were modeled, often in an axisymmetric framework. The configuration for these simulations was typically guided by previous laboratory modelings (Ward 1972). Laboratory research and idealized modeling have indicated that 
The first three-dimensional (3D) simulations of deep moist supercellular convection conducted in the mid-/late 1970s were run over limited domains and were coarsely resolved because of the computational limitations of the time. Klemp and Wilhelmson (1978a,b) and Wilhelmson and Klemp (1978) utilized a grid spanning $24 \times 24 \times 20$ $(I I, 520)$ grid points in three-dimensional simulations of supercells, with horizontal grid spacings of $2 \mathrm{~km}$ (Klemp and Wilhelmson 1978a) and a domain extending to only $10 \mathrm{~km}$ in the vertical. At this resolution, computational stability could be maintained at time steps on the order of $10 \mathrm{~s}$ and simulations were carried out for about an hour of simulation time. Up through the 1990s, many simulations of supercells continued to be run at horizontal gridpoint spacings of $\mathrm{I} \mathrm{km}$ but with larger domains to contain the storm and its surrounding environment.

Near the turn of the century, supercomputers had evolved toward massively parallel systems, with an inexorable transition to distributedmemory architectures. Message passing libraries such as Message Passing Interface (MPI) were developed to facilitate the exchange of data between sharedmemory nodes on such machines. The clock speed of individual floating-point processor units continued to increase, following “Moore's law” (Moore 1965), and the amount of memory addressed by each unit increased as well. These massively parallel, distributed-memory supercomputing architectures presented new opportunities for scientists, allowing for models with increasing complexity and sophistication that were really only constrained by their ability to scale efficiently when utilizing tens of thousands of computing cores. The reward for this effort has been the ability to run much larger simulations than ever before, covering more grid points at higher resolution, whether at global, regional, or cloud scales.

Models exploiting modern supercomputers can produce a staggering amount of data. We are utilizing the
Blue Waters supercomputer (Bode et al. 2013; Kramer et al. 2014), which became operational at the National Center for Supercomputing Applications in 2013. Blue Waters is a massively parallel computer containing over 22,500 compute nodes, each of which contains 16 floating-point processing cores and $64 \mathrm{~GB}$ of memory. The simulation reported herein was carried out on a grid of $2,200 \times 2,200 \times 380$ (about 160,000 times more grid points than the early simulations noted above) with 30-m grid spacing in all directions in the inner part of the domain and produced around 100 TB of model output when saving the data frequently. Computational stability was guaranteed using a 0.2-s time step and it took about I5 h of wall clock time to simulate an hour of cloud time. More recent 20-m simulations conducted by our group (currently being analyzed) on Blue Waters produced over 0.5 petabytes of data. Such large amounts of data create significant challenges regarding analysis, visualization, and archiving. tornadoes may take on a range of kinematic structures. These include single vortices exhibiting positive vertical velocity throughout (single cell), single vortices exhibiting a downdraft within the tornado core (two cell), and multiple vortices rotating about the center of broader circulation [for an overview of idealized modeling research, see Rotunno (2013)].

As computing technology has advanced, it has become possible to run simulations of supercells at resolutions where tornadoes are explicitly resolved. Xue (2004) used the 1977 Del City supercell environment (Klemp et al. 1981) to initialize the Advanced Regional Prediction System (ARPS) model (Xue et al. 2003) at 25-m horizontal grid spacing. The simulated supercell produced a tornado with winds exceeding $120 \mathrm{~m} \mathrm{~s}^{-1}$ at the surface; however, in order to run at this resolution, a small $\left(50 \times 50 \mathrm{~km}^{2}\right)$ domain was employed and the simulation suffered from nonphysical interaction with model's 


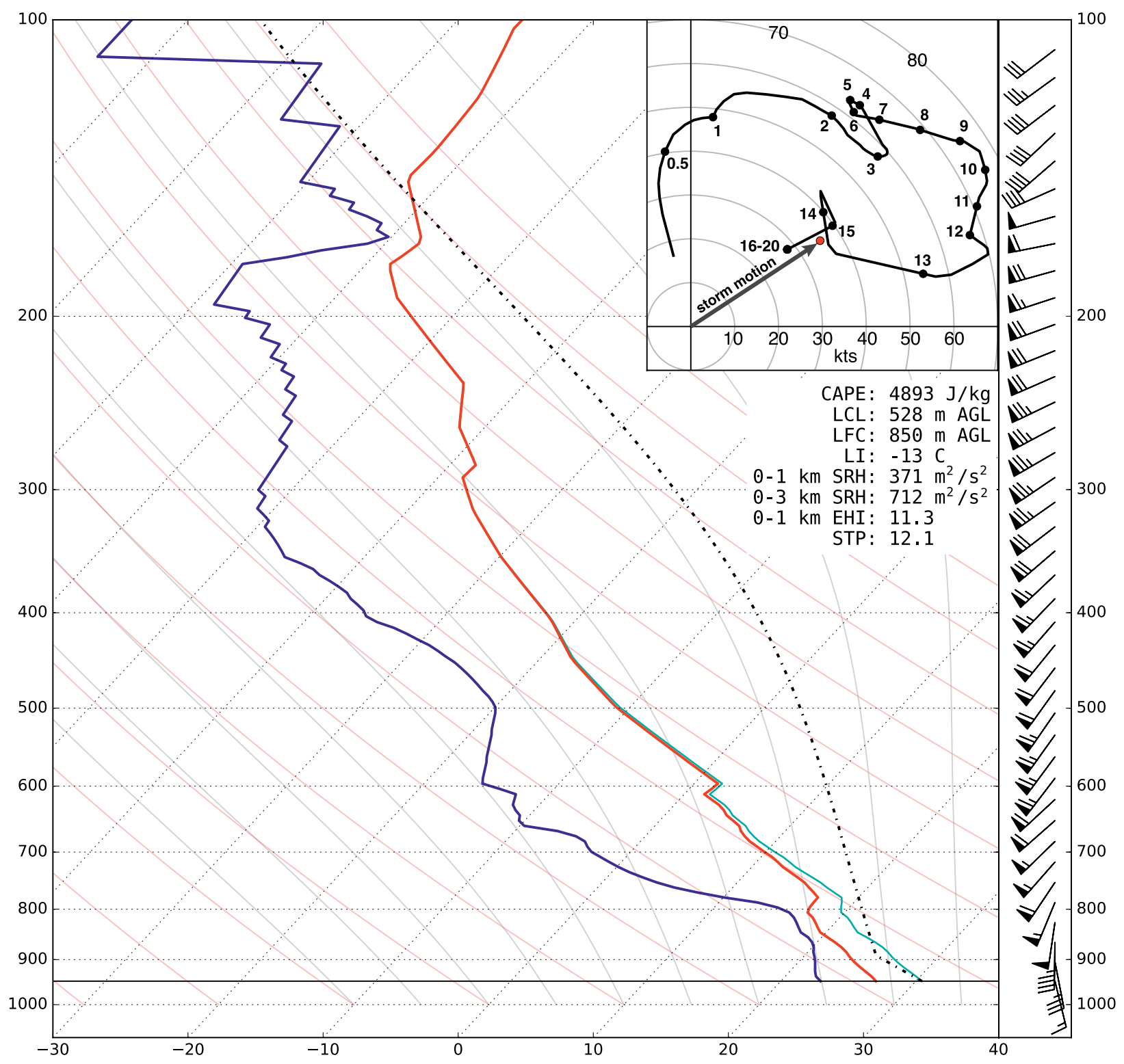

FIG. I. The sounding and hodograph, taken from a I-h RUC forecast off the right flank of the supercell that produced the 24 May 20 II long-track EF5 tornado near EI Reno, Oklahoma. Significant levels were calculated based on surface-based parcel ascent (as shown), with CAPE calculated using virtual temperature. Wind barbs and hodograph values are shown in nautical miles per hour (full barb $10 \mathrm{kt}$; I kt $=0.5 \mathrm{l} \mathrm{m} \mathrm{s}^{-1}$ ). Hodograph levels are specified in $\mathbf{k m}$.

lateral boundaries. More recently, Schenkman et al. (2014) simulated a tornadic supercell using ARPS in which the tilting of frictionally generated horizontal vorticity was cited as an important source of nearground vertical vorticity. In Schenkman et al. (2014) and Xue et al. (2014), Weather Surveillance Radar-1988 Doppler (WSR-88D) data of the 8 May 2003 Oklahoma City tornadic supercell were assimilated into ARPS, which produced a tornadic supercell that generally agreed with observations. In both simulations, a series of nested grids were utilized where the finest mesh, centered upon the supercell, employed 50-m grid spacing.
Recently our team modeled a tornado in an idealized supercell simulation with 30-m grid spacing on the Blue Waters supercomputer (Bode et al. 2013; Kramer et al. 2014) utilizing Cloud Model 1 (CM1; Bryan and Fritsch 2002). The tornado evolved from a narrow single-celled tornado to a 800-m-wide, rain-wrapped, wedge-shaped, two-celled tornado that exhibited storm-relative winds as high as $143 \mathrm{~m} \mathrm{~s}^{-1}$ and maintained winds in excess of $90 \mathrm{~m} \mathrm{~s}^{-1}$ (the EF5 threshold) for 38 uninterrupted minutes. The total life span of the tornado was nearly $2 \mathrm{~h}$. Utilizing state-of-the-art visualization and analysis tools, we created animations (video imagery) of the 


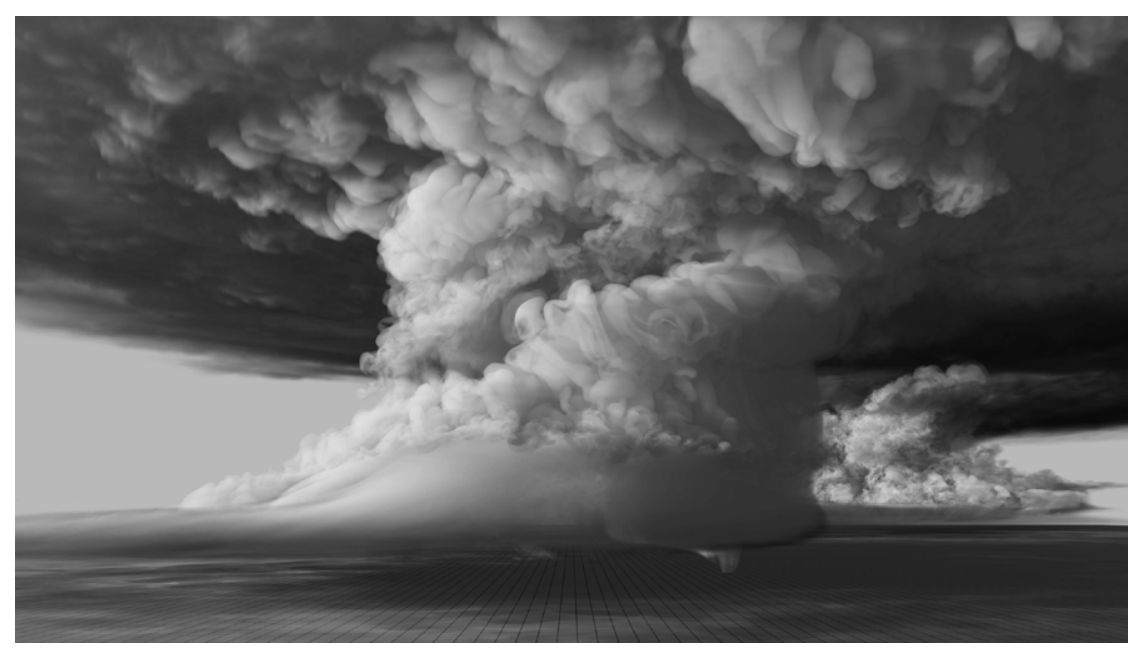

FIG. 2. Photorealistic rendering of the supercell cloud field (looking north) at $t=7,200$ s from a height of $3.7 \mathrm{~km}$ AGL. Image courtesy of David Bock. phenomena (Bryan and Fritsch 2002). A summary of CM1 configuration options is found in Table 1 . The sounding and hodograph used to initialize the model (Fig. 1) was taken from a Rapid Update Cycle (RUC; Benjamin et al. 2004) model 1-h forecast, off the right flank of the storm that spawned a long-lived EF5 tornado on 24 May 2011, in central Oklahoma [for descriptions of the observed storms refer to Tanamachi et al. (2015), Houser et al. (2015), and French et al. (2015)]. The base-state environment is storm at very high temporal and spatial resolution in order to capture the flow features, some highly transient, found within tornadic supercells (animations of the simulation can be viewed in the online supplement at http://dx.doi.org//0.II75/BAMS-D-15-00073.2). This paper serves two purposes: first, to provide an overview of the methods chosen to efficiently write, organize, and visualize over $100 \mathrm{~TB}$ of model data (see "Computational challenges, past and present" and "Visualization and animation" sidebars) and, second, to describe salient features of the simulation and compare aspects of the modeled tornadic supercell to observations. Some important structural and process aspects that appear essential to tornado development and evolution within the modeled storm are identified, but thorough analysis of these features is beyond the scope of this paper and will be described in future publications.

\section{MODEL CONFIGU. RATION AND BASE- STATE ENVIRONMENT.}

The model used for this study, CM1 version 16 , is a threedimensional, nonhydrostatic cloud model designed for idealized studies of atmospheric

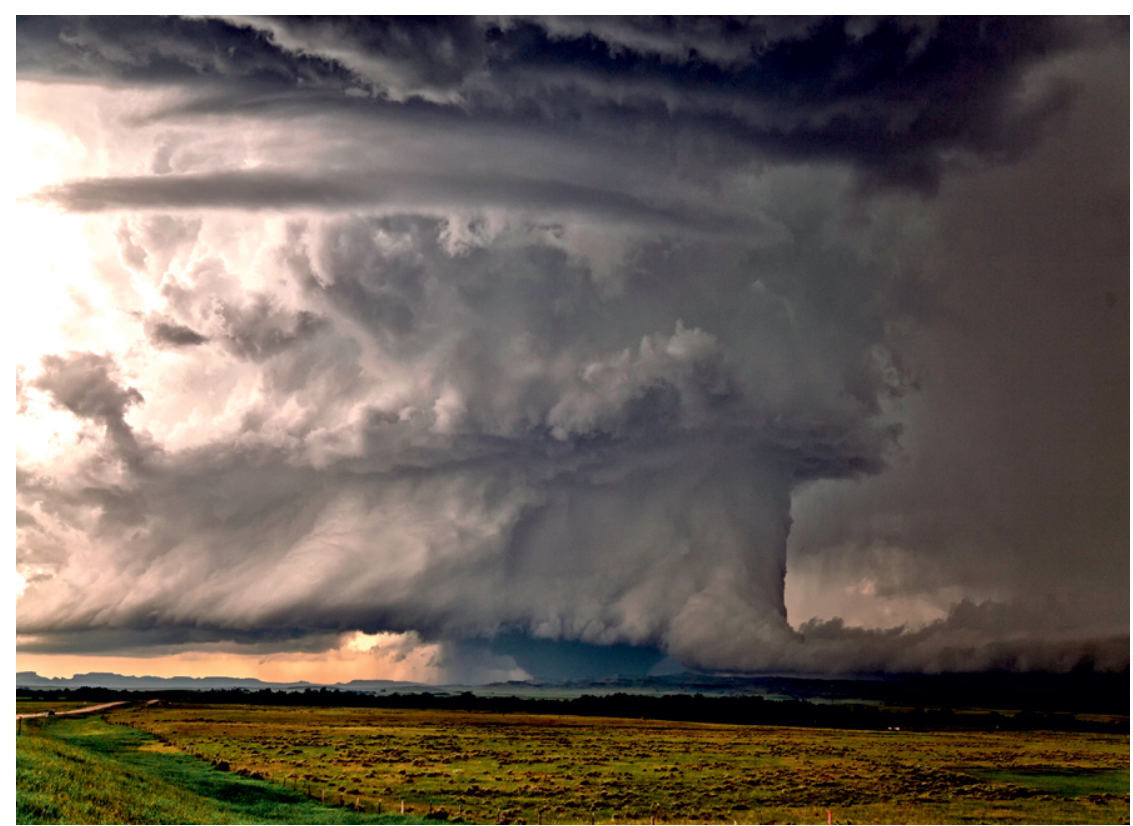

FIG. 3. A tornadic supercell over Carter County, Montana, on 17 Jun 2014, looking toward the west. A large and long-lived tornado may be seen to the rear of where an inflow cloud band along a forward-flank boundary is moving into the storm updraft. Cloud motions within this persistent inflow band from videography were consistent with an SVC. Photograph courtesy of Roger Hill. 
correlated with significant tornadoes (Rasmussen and Blanchard 1998; Rasmussen 2003; Thompson et al. 2003). The LCL (528 $\mathrm{m}$ ) and $0-1-\mathrm{km} \mathrm{SRH}\left(371 \mathrm{~m}^{2} \mathrm{~s}^{-2}\right)$ for this environment lie in the lower/upper (respectively) $10 \%$ of the distributions for supercells producing significant tornadoes (Thompson et al. 2003). Composite parameters such as the $0-1-\mathrm{km}$ energy helicity index (11.3; Hart and Korotky 1991; Rasmussen 2003) and the significant tornado parameter (12.1) also lie in the upper $10 \%$ of environments that support significant tornadoes (Thompson et al. 2003).
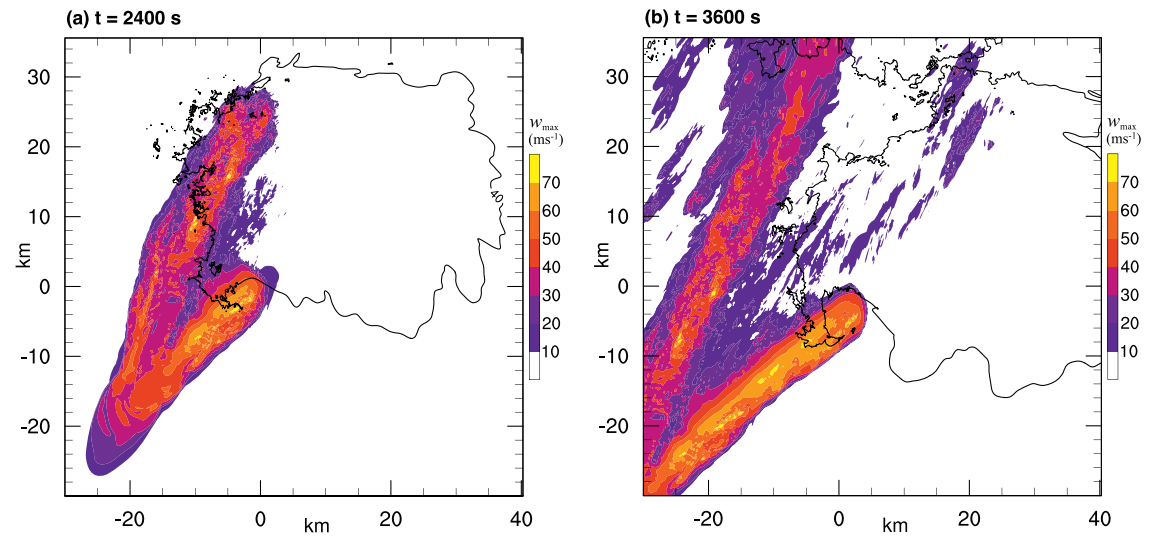

FIG. 4. (a) Updraft "swath" (history of maximum w) at $5 \mathrm{~km}$ AGL, evaluated every model time step, translated with the moving domain (color-filled contour), and the $500 \mathrm{~m}$ AGL $40-\mathrm{dBZ}$ reflectivity contour at $t=2,400 \mathrm{~s}$. The image covers a $70 \mathrm{~km} \times 70 \mathrm{~km}$ region centered upon the updraft of the rightmoving storm. The storm has split into a left mover with approximate updraft centroid located at $(0 \mathrm{~km}, 25 \mathrm{~km})$ and a right mover, the storm that is the subject of this paper, located near $(0 \mathrm{~km}, 0 \mathrm{~km})$. (b) As in (a), at $t=3,600 \mathrm{~s}$, at which point the left mover has exited the subdomain covered by the image. The location of the updraft at $5 \mathrm{~km}$ coincides with the weak echo region (WER) in the reflectivity field at $500 \mathrm{~m}$.
RESULTS. A natural ini-

tial question is, how does this simulation compare to the El Reno tornadic supercell of 24 May 2011? While we do not claim we are modeling the El Reno event, given the heterogeneous influences on the real supercell in contrast to the homogeneous model initialization, the storms do share some common characteristics. The supercells are relatively long lived with the modeled storm exhibiting supercell structure for $2.5 \mathrm{~h}$ (still present at the simulation termination) while the actual El Reno storm displayed supercell characteristics for roughly $3 \mathrm{~h}$ before its identity became convoluted in an evolving line of storms. While both actual (National Weather Service 2011b) and modeled (see "Storm structure prior to tornadogenesis") storms displayed classic supercell reflectivity structure (Moller et al. 1994) over extended periods, the actual storm structure was substantially influenced by numerous cell mergers that were not present in the simulation (National Weather Service 2011b; Tanamachi et al. 2015). Of note, the El Reno supercell produced a family of tornadoes, with the strongest one designated herein as the El Reno tornado. Given computational constraints, this simulation was not run long enough to see if the modeled storm would also produce a series of tornadoes. Both actual and modeled tornadoes were of EF5 intensity ${ }^{1}$ and

\footnotetext{
${ }^{1}$ Although the EF scale is based upon damage and references ground-relative winds, the modeled tornado produced both storm- and ground-relative winds associated with the EF5 range.
}

long lived, with the El Reno tornado lasting $105 \mathrm{~min}$ (National Weather Service 2011a) compared with the modeled tornado lifespan of $118 \mathrm{~min}$. The El Reno tornado had a $101-\mathrm{km}$ pathlength compared with its model counterpart's $120 \mathrm{~km}$.

The simulated storm takes on physically realistic characteristics (Fig. 2) in the volume-rendered hydrometeor fields (Orf et al. 2016) that exhibit many salient visible features associated with observed supercells (Fig. 3), including a well-defined wall cloud with an inflow band, and a laminar cloud structure at low levels that transitions into a convective regime at mid- and upper levels. An animation of the simulated cloud and precipitation fields also shows features routinely observed, such as periodic rain curtains that wrap around the low-level mesocyclone/tornado and a tornado that becomes more rain wrapped in time.

Storm structure prior to tornadogenesis. Following initialization, the storm rapidly evolves into a supercell, and by $2,400 \mathrm{~s}$ the storm has split, with the right mover beginning to exhibit a persistent, strong updraft at $5 \mathrm{~km}$ and a nascent hook echo at $500 \mathrm{~m}$ AGL (Fig. 4a). At this time, the left member from the storm split is much weaker and less organized, consistent with previous numerical studies that showed enhancement of the right mover in environments with hodographs turning clockwise with height (Klemp and Wilhelmson 1978b), as is the case here. By $t=3,600 \mathrm{~s}$, the right-moving storm has become 


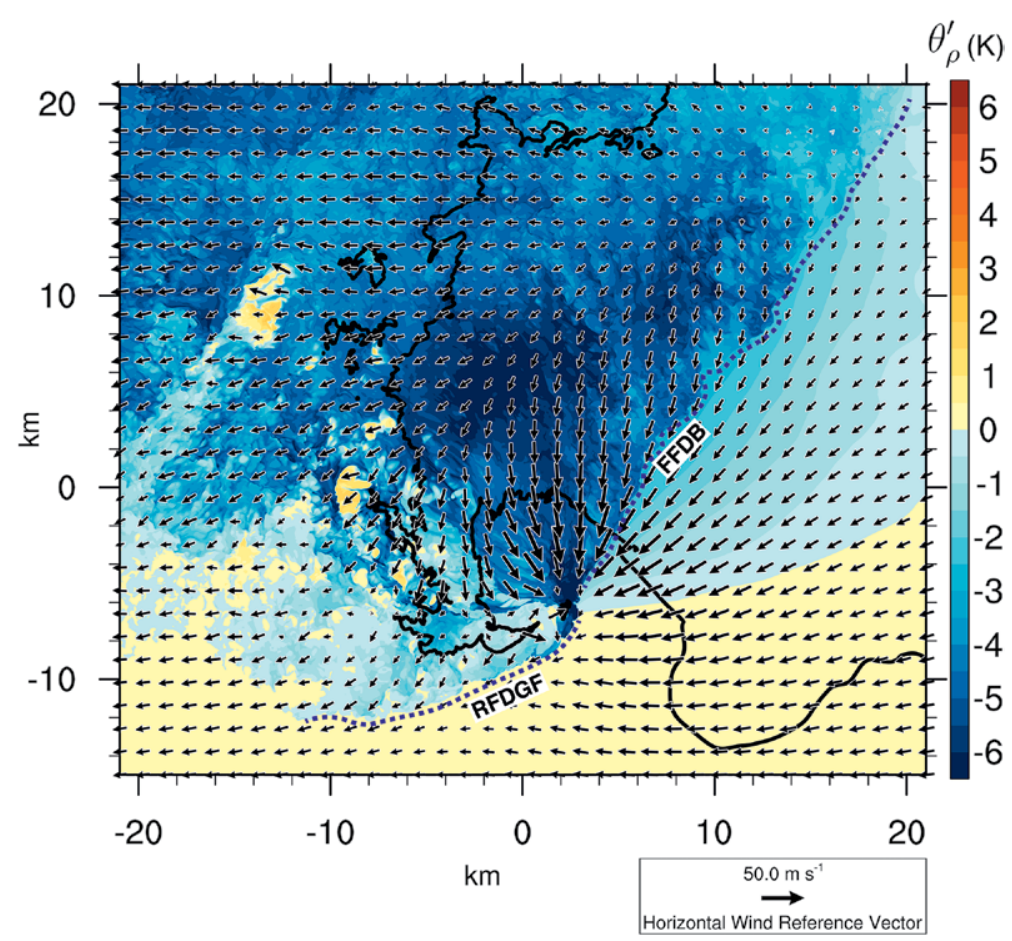

FIG. 5. 500-m AGL 40-dBZ reflectivity contour (thick black line), surface $\theta_{\rho}^{\prime}$ (color-filled values in intervals of $0.5 \mathrm{~K}$ ), and storm-relative surface wind vectors drawn every 40th grid point (every $1.2 \mathrm{~km}$ ) at $t=3,600 \mathrm{~s}$. this boundary is generally characterized by maximum $\theta_{\rho}^{\prime}$ deficits of less than $2 \mathrm{~K}$. Observational research has shown that tornado occurrence and intensity are more likely with RFD outflows having only small negative buoyancy (Markowski et al. 2002; Grzych et al. 2007; Lee et al. 2012). Surface storm-relative wind vectors show pronounced convergence indicative of a very strong updraft near the intersection of the RFDGF and FFDB (Fig. 5).

Tornadogenesis. Tornado development in the simulation is associated with the evolution of several concomitant components of the parent storm. At $t=3,600 \mathrm{~s}$, the RFDGF is discernible in the $\theta^{\prime}$ field (Fig. 6a), but within $1 \mathrm{~km}$ west of the boundary, $\theta_{\rho}^{\prime}$ deficits nearly vanish. Only weak storm-relative outflow is seen behind the RFDGF. A few kilometers to the northwest, an RFD internal surge (RFDIS) (Finley and Lee 2004; Lee et al. 2004, 2011, increasingly separated from the left member and exhibits a more pronounced hook (Fig. 4b). By this time, the cold/cool pools have spread out from the rear- and forward-flank downdrafts (see Fig. 5). ${ }^{2}$

The southern portion of the storm's forwardflank region is characterized by a relatively smooth northwest-southeast $\theta_{\rho}^{\prime}$ gradient and, like some other observed and modeled supercells, the wind field changes very little across this region (Beck and Weiss 2013). To the northwest of this region, a prominent kinematic and thermodynamic boundary (indicated by a thick dashed line labeled FFDB in Fig. 5) is readily identified. This boundary persists throughout much of the simulation and is the effective forward-flank downdraft boundary (FFDB). The rear-flank downdraft (RFD) gust front is indicated by a dashed blue line labeled RFDGF in Fig. 5 . Following the development of the RFD, the simulated storm acquires a "horseshoe-shaped" updraft prior to tornadogenesis similar to observations (Lemon and Doswell 1979). The outflow immediately west of
2012; Marquis et al. 2012; Kosiba et al. 2013; Skinner et al. 2014, 2015; Schenkman et al. 2016) is apparent in the kinematic field with markedly stronger northerly/ northwesterly storm-relative winds. During the period from $t=3,600$ to $4,200 \mathrm{~s}$, somewhat more negatively buoyant air associated with multiple RFDISs sweeps southeastward. The RFDISB shown in Fig. $6 \mathrm{~b}$ is an augmentation of the surge boundary in Fig. $6 \mathrm{a}$ after being reinforced by two successive surges. This boundary ultimately merges with the RFDGF around 4,500 s.

By $t=5,100 \mathrm{~s}$, the leading edge of the RFDGF is characterized by a sharp $\theta_{\rho}^{\prime}$ gradient backed by widespread storm-relative southeastward flow (Fig. 6c). At this time, a bulge in the leading edge of the storm's cold pool has formed, flanked by a cyclonic/anticylonic vortex pair. The southern member of this vortex couplet will form into a narrow anticyclonic tornado, while the northern cyclonic vortex, already at tornadic intensity, will grow and intensify, becoming the longtrack EF5 tornado. Figure $6 \mathrm{c}$ also indicates that regions of marked surface ${ }^{3}$ streamwise vorticity (regions

\footnotetext{
${ }^{2}$ In Figs. 5 and 6, fluctuations in the density potential temperature $\left(\theta_{\rho}\right)$ (Emanuel 1994) from a base-state value are used to thermodynamically characterize the RFD and forward-flank downdraft (FFD) cold pools, as buoyancy is proportional to density potential temperature perturbation $\left(\theta_{\rho}^{\prime}\right)$.
}

${ }^{3}$ For the sake of brevity, we refer to the surface as the lowest scalar model level, which is $15 \mathrm{~m} \mathrm{AGL}$, and we refer to the horizontal components of vorticity interpolated to the model's lowest scalar vertical level (15 m AGL) as the surface horizontal vorticity. 


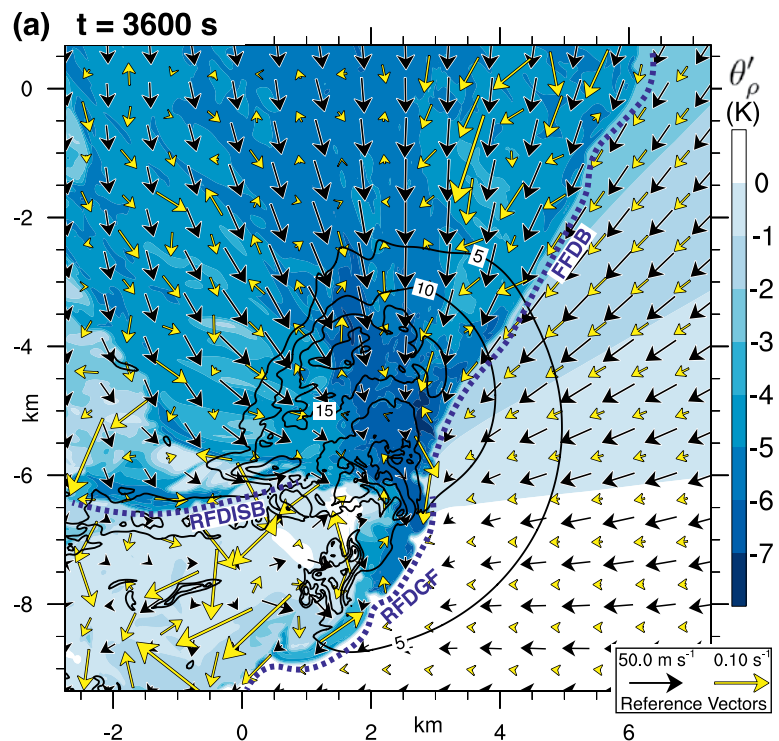

(c) $t=5100 \mathrm{~s}$

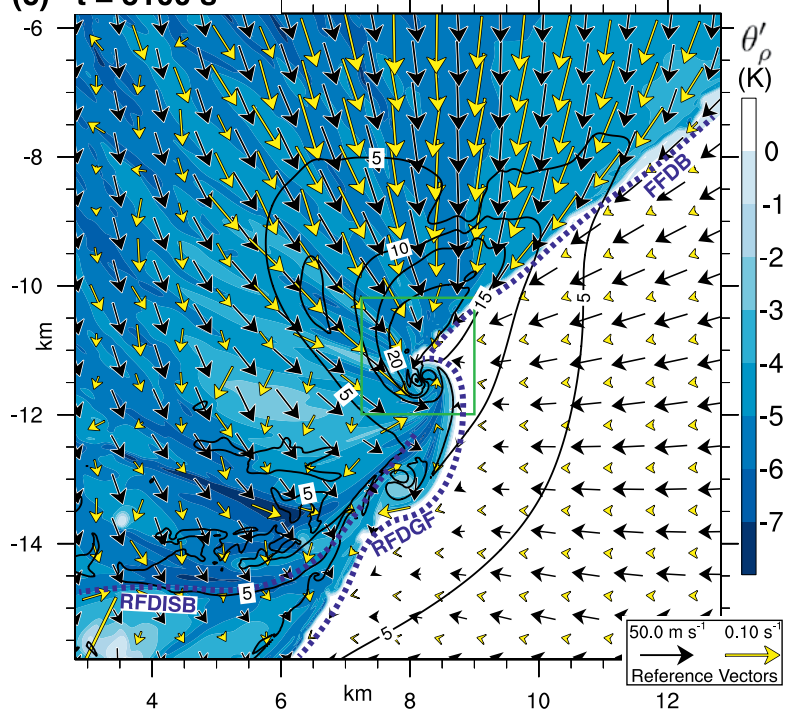

(b) $t=4200 s$

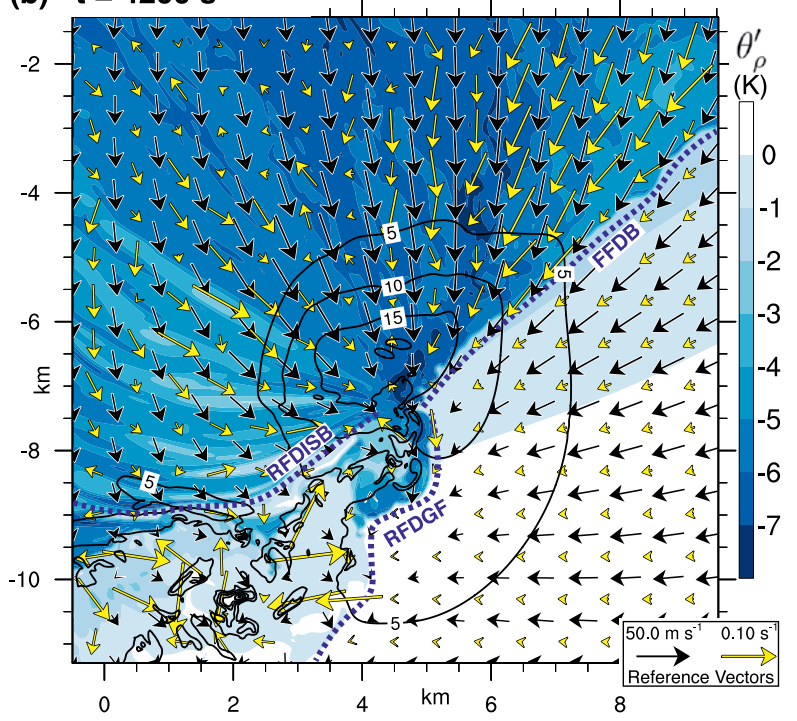

(d) $\mathbf{t}=\mathbf{5 1 0 0 \mathrm { s }}$

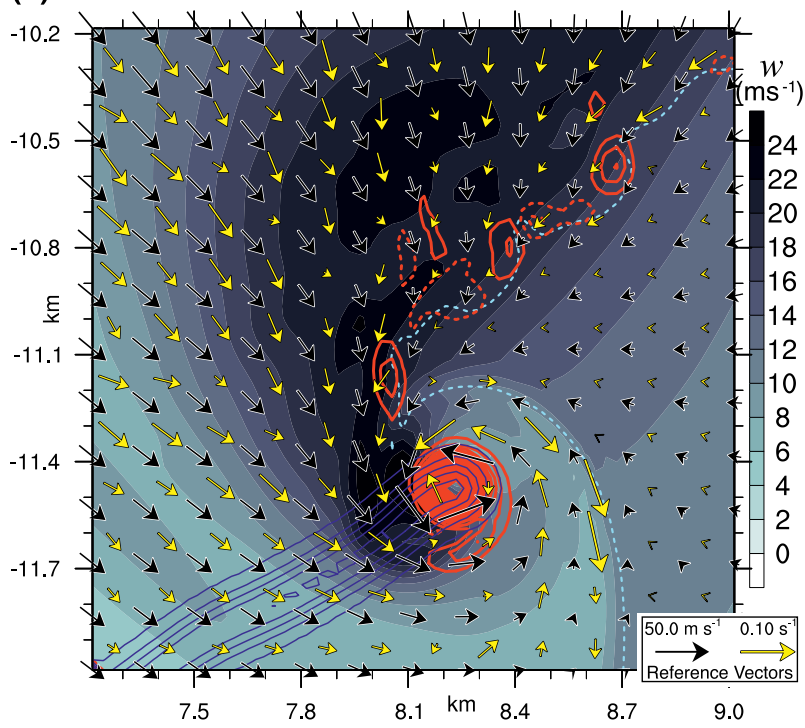

Fig. 6. (a) At $t=3,600 \mathrm{~s}$, surface cold pool negative $\theta_{\rho}^{\prime}$ (color-filled contour plotted every I K), positive $w$ component of the wind at $495 \mathrm{~m}$ AGL (solid contour plotted every $5 \mathrm{~m} \mathrm{~s}^{-1}$ ), surface storm-relative horizontal wind vectors plotted every 22 grid points (black vectors), surface horizontal vorticity vectors plotted every 22 grid points (yellow vectors). (b) As in (a), but at $t=4,200 \mathrm{~s}$. (c) As in (a), but at $t=5,100 \mathrm{~s}$. (d) At $t=5,100 \mathrm{~s}$, zoomed in on region surrounding tornado [inset region indicated by green box in (c)]: positive $w$ component of the wind $495 \mathrm{~m}$ AGL (color-filled contour, every $2 \mathrm{~m} \mathrm{~s}^{-1}$ ), surface cold pool boundary indicated by the -I-K $\theta^{\prime}$ contour (dashed light blue line), surface negative pressure perturbation swath (time history of minimum surface pressure, relative to the ground) contoured every $10 \mathrm{hPa}$ (solid blue line), surface vertical component of vorticity ( $\zeta$; red contours plotted every $0.1 \mathrm{~s}^{-1}$ starting at $-0.15 \mathrm{~s}^{-1}$, with dashed negative values), surface horizontal wind and horizontal vorticity vectors plotted every 5 grid points.

where the velocity and vorticity vectors are aligned) have expanded and intensified from sectors within the forward flank and in RFD surges (Figs. 6a,b) to a much broader region arcing from the FFDB through the rear flank west of the surface circulation. This streamwise vorticity is converging toward the developing tornado (Fig. 6d) within a region of very strong low-level updraft (maximum values exceeding $24 \mathrm{~m} \mathrm{~s}^{-1}$ at $495 \mathrm{~m} \mathrm{AGL}$ ).

The flow field along the FFDB possesses substantial vertical vorticity (broad regions with values from 0.04 to $0.08 \mathrm{~s}^{-1}$ ) along the erect portion of the boundary. In effect, this region is a vertical vorticity sheet (VVS). While the sense of the horizontal shear across 


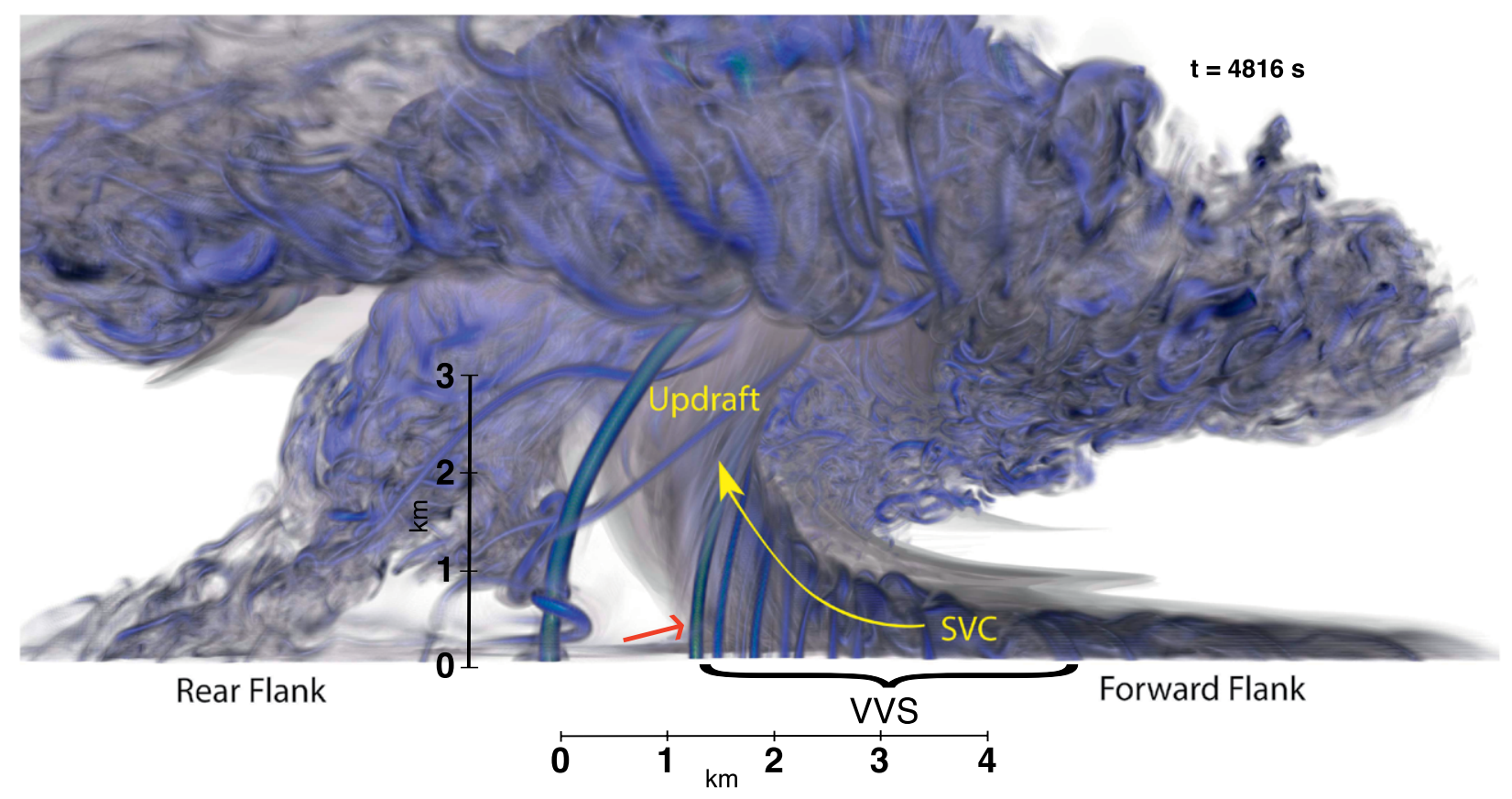

FIG. 7. Volume-rendered vorticity magnitude with a lower threshold of $0.025 \mathrm{~s}^{-1}$ at $t=4,816 \mathrm{~s}$, prior to tornado formation. View is looking north. SVC marks the feature we call the streamwise vorticity current, and VVS marks the location of the vertical vorticity sheet. The red arrow points to the vortex that becomes the tornado. The yellow arrow indicates the storm-relative path of the air within the streamwise vorticity current as it is drawn into the updraft.

the boundary is supportive of a band of cyclonic vertical vorticity lying along it, the local character of the vertical vorticity structure is very complex. Much of the local vertical vorticity along the FFDB results from patches and linear segments of vertical vorticity of both signs that move toward the boundary from within the forward-flank outflow. Thus, unlike a more symmetric idealized vortex sheet that breaks down into same-signed vortices by horizontal shearing instability [HSI; see Batchelor (1967)] with a preferred wavelength (Miles and Howard 1964), there are both cyclonic and anticyclonic vortices or vorticity patches present along the boundary, as shown in Fig. $6 \mathrm{~d}$ and subsequent figures. The FFDB represents a VVS where cyclonic vortices and vorticity patches are favored but not exclusive. The potential role that sheets of vertical vorticity play in supercell tornadogenesis has been previously suggested (e.g., Brandes 1977, 1978; Lee and Wilhelmson 1997) with more recent interest in these sheets as in Finley et al. (2002), Bluestein et al. (2003), Finley and Lee (2004), Gaudet et al. (2006), Lee et al. (2012), Markowski et al. (2014), and Dahl et al. (2014). A more in-depth study of vertical vorticity along the FFDB will be presented in a subsequent paper.

A train of vortices within the VVS (Fig. 7) move in a southwestward storm-relative direction consistent with the mean velocity vector along the FFDB. Similar predominantly cyclonic misocyclone-scale vortices moving down boundaries along/within the forward flank of supercells that appear to merge into the lowlevel mesocyclone or tornado cyclone have also been observed in mobile Doppler radar data (e.g., Snyder et al. 2013; Wurman and Kosiba 2013; Wurman et al. 2014). Evidence of discrete vorticity patches (and associated negative pressure perturbations) merging with the surface circulation center just prior to tornadogenesis is also seen in some multi-Doppler analyses (e.g., Dowell et al. 2002; Markowski et al. 2012a,b). In our simulation, the development of an inflection point along the FFDB serves as a focus point for the accumulation of cyclonic vorticity moving rearward from the VVS. A more detailed view of the genesis of the embryonic cyclonic vortex that will eventually become dominant is shown in Fig. 8. From $t=4,444$ to $4,488 \mathrm{~s}$, a cyclonic vortex (identified with black arrows) is visible within a collection of cyclonic and anticylonic vortices near the growing boundary infection point. By $t=4,628 \mathrm{~s}$, both the cyclonic vortex and an adjacent anticylonic vortex have strengthened while more vortices originating along the FFDB move rearward, where they either merge with the developing tornado (cyclonic-cyclonic interaction) or are swept around the developing tornado and upward 


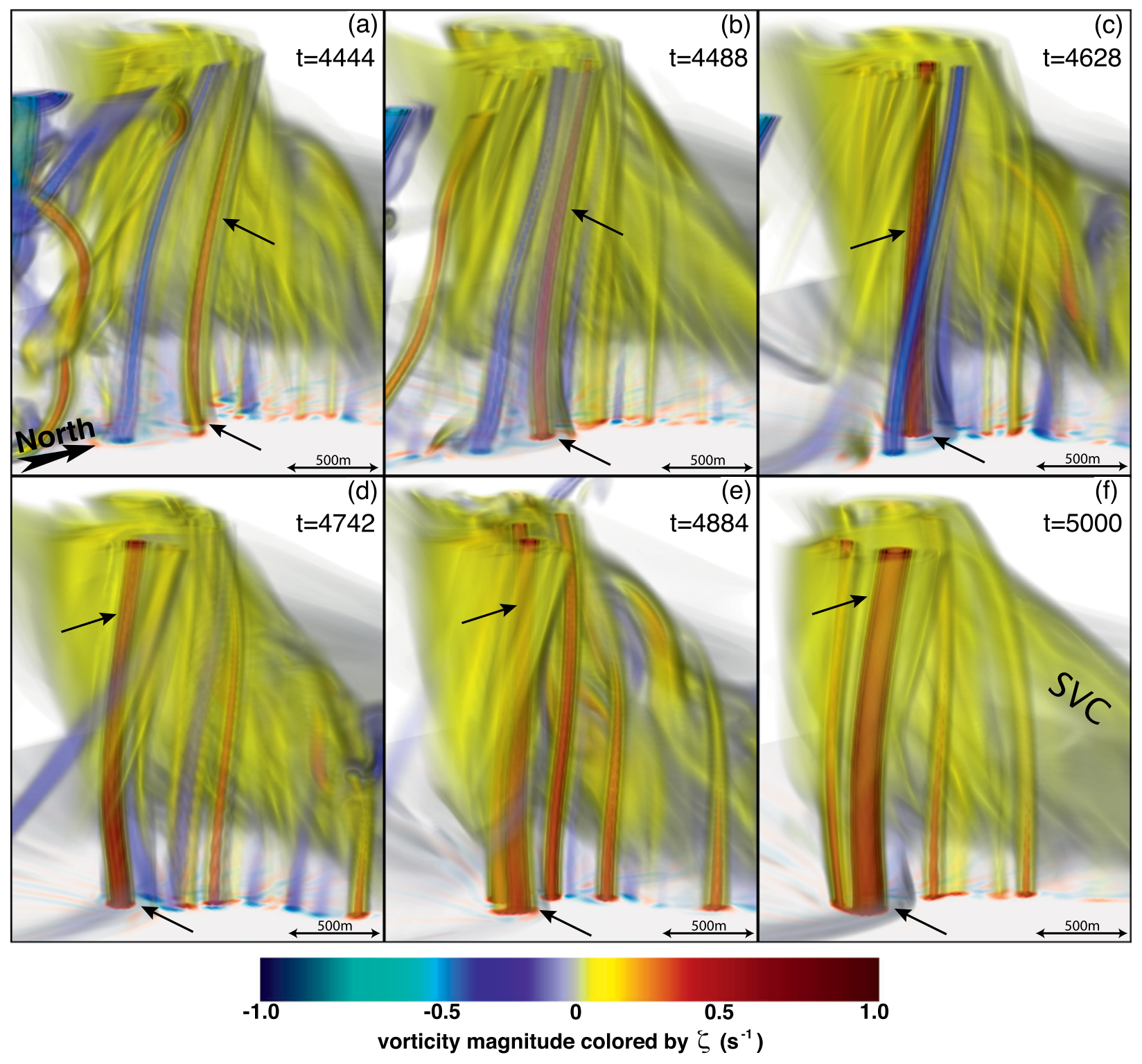

FIG. 8. Volume-rendered vorticity magnitude colored by the vertical component of vorticity $\left(\zeta, \mathrm{s}^{-1}\right)$. Values of vorticity magnitude less than $0.025 \mathrm{~s}^{-1}$ are not shown. View is looking northwest, and the vorticity field is clipped at $2 \mathrm{~km}$ AGL. Black arrows indicate the location of the cyclonic vortex that becomes the long-track tornado.

(cyclonic-anticyclonic interaction). At $t=4,884 \mathrm{~s}$, a diffuse region of cyclonic vorticity is observed to surround the vortex aloft, while three cyclonic vortices are moving southwestward along the FFDB. By $t=4,950 \mathrm{~s}$, the tornado, producing instantaneous storm-relative winds up to $48 \mathrm{~m} \mathrm{~s}^{-1}$, has widened and continues to strengthen over time (Fig. 9).

Temporal and spatial aspects of tornadogenesis appear dependent upon the evolution of a strong updraft aligned over the low-level features. Updraft intensification in the simulation seems related to the development of a flow field feature we call a streamwise vorticity current (SVC) that preceded tornadogenesis. The SVC is a persistent "tube" of streamwise vorticity located along the FFDB that flows rearward along the FFDB and eventually upward into the updraft. It is confined to a region immediately on the cool side of the FFDB where streamwise horizontal vorticity can be generated through baroclinic effects. Figures 7 and 8 depict the location and orientation of the SVC during the early stages of tornadogenesis, and Fig. 10 presents three-dimensional streamwise vorticity shortly following tornadogenesis. The SVC bears some resemblance to the rotor simulated by Schenkman et al. (2012) but the SVC extends deeper into the forward flank and, unlike the rotor, the SVC vorticity cannot be attributed to surface friction owing to the free-slip lower boundary condition. 


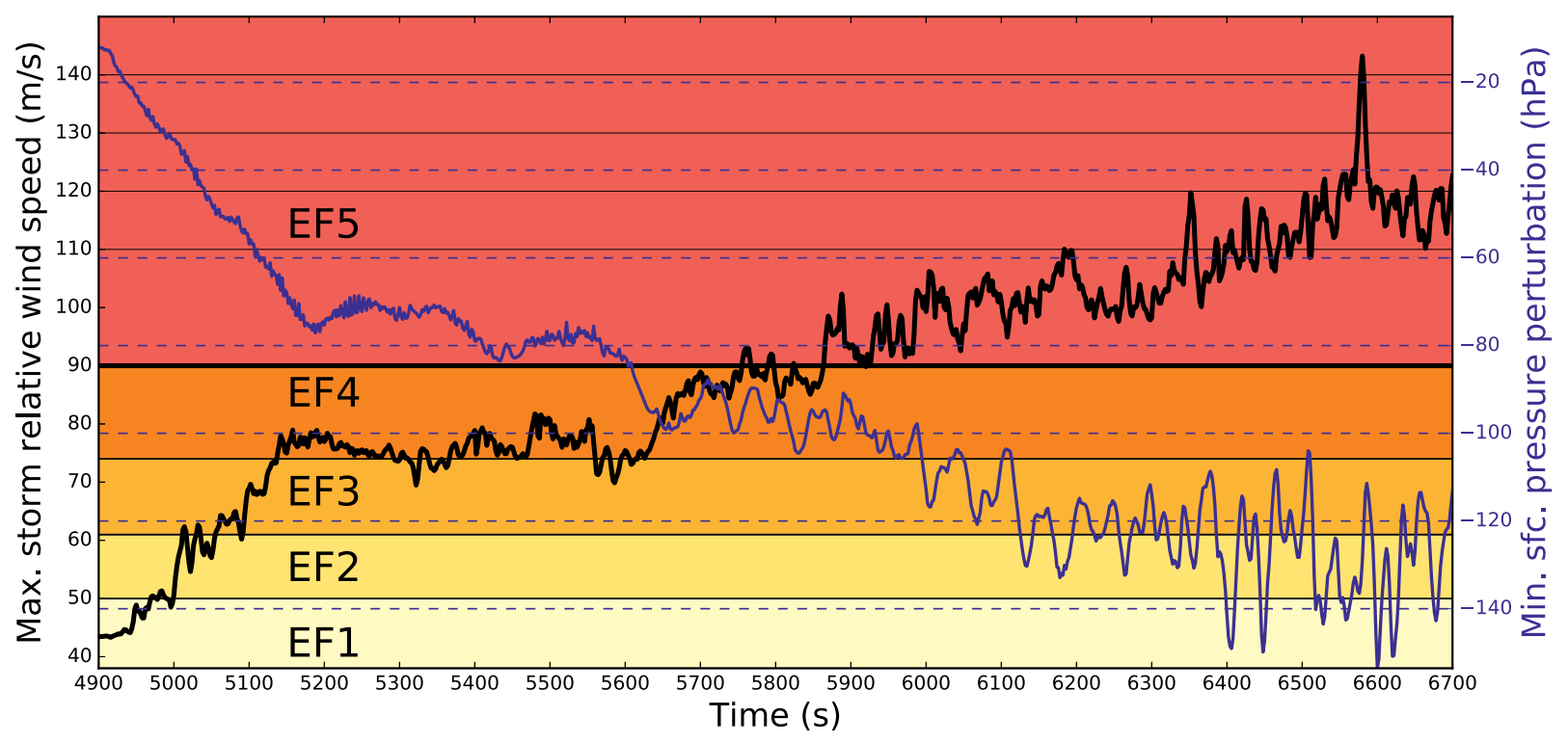

FIG. 9. Time series of maximum storm-relative surface wind speed (black line) and minimum surface pressure perturbation (blue line) associated with the tornado, sampled every $2 \mathrm{~s}$, for the first $1,800 \mathrm{~s}$ of its duration (during genesis and early portion of mature stage). Enhanced Fujita wind speed ranges are indicated by color for reference, with the thick black line indicating the EF5 threshold.

Prior to the SVC being tilted into the updraft, horizontal vorticity within the SVC is generated within the buoyancy gradient associated with the FFDB (Fig. 6). The SVC strengthens as the FFDB buoyancy gradient sharpens. In the approximate 10-12 min before tornado development, the SVC markedly intensified. As the SVC strengthens, tilting imparts increasing rotation to the low-level mesocyclone and concurrent intensification of the low-level updraft ensues. This updraft strengthening is consistent with a strong upward-directed, rotationally induced perturbation pressure gradient acceleration. The resultant convergence beneath this strengthening updraft can be inferred from Fig. 6. Thus, the timing and location of tornadogenesis appear strongly associated with the intensification and location of abrupt tilting of the SVC. The resulting updraft alignment over the surface features appears related and crucial to the process (Dowell and Bluestein 2002; Marquis et al. 2012; Skinner et al. 2014). It is worth noting that SVC strength is also likely influenced by feedbacks within the system. As the low-level updraft strengthens as a result of rotationally induced upward perturbation pressure gradient forcing as just described, accelerating inflow would subject the SVC to horizontal vorticity stretching and resultant intensification.

On some occasions, rotation associated with the SVC can be seen manifest in inflow cloud band motions aligned along the FFDB or internal forwardflank boundary. As an example, SVC-related motion was apparent within the inflow cloud band that extended northeast from the main updraft in the 17 June 2014 Carter County Montana tornadic supercell (Fig. 3) as evidenced in videography.

Unlike the flow depicted in historical prominent works such as Rotunno and Klemp (1985), where parcels arriving at the low-level mesocyclone vertical vorticity maximum (250-m level) first descended on the cool side of the FFDB to low levels before rising sharply, the flow of air within the SVC slowly ascends in a helical pattern along the FFDB until being tilted abruptly upward within the lowest kilometer (Figs. 7 and 10). Thus, the SVC supports a deep and intense mesocyclone with the lower reaches of that support at an altitude normally associated with the low-level mesocyclone.

During tornadogenesis, the simulated vortex intensifies nearly simultaneously from the surface through the storm midlevels similar to the El Reno tornado analyzed from mobile Doppler radar data (Houser et al. 2015). This characteristic for the vortex to intensify nearly concurrently over a deep layer is also consistent with the modeling results of Trapp and Fiedler (1995) and Trapp and Davies-Jones (1997), and perhaps additionally, consistent with some of the cases identified by Trapp et al. (1999) within their nondescending tornadic vortex signature categorization. Moreover, the recent analysis of French et al. (2013) utilizing high-temporal-resolution mobile Doppler radar data indicates that nondescending 


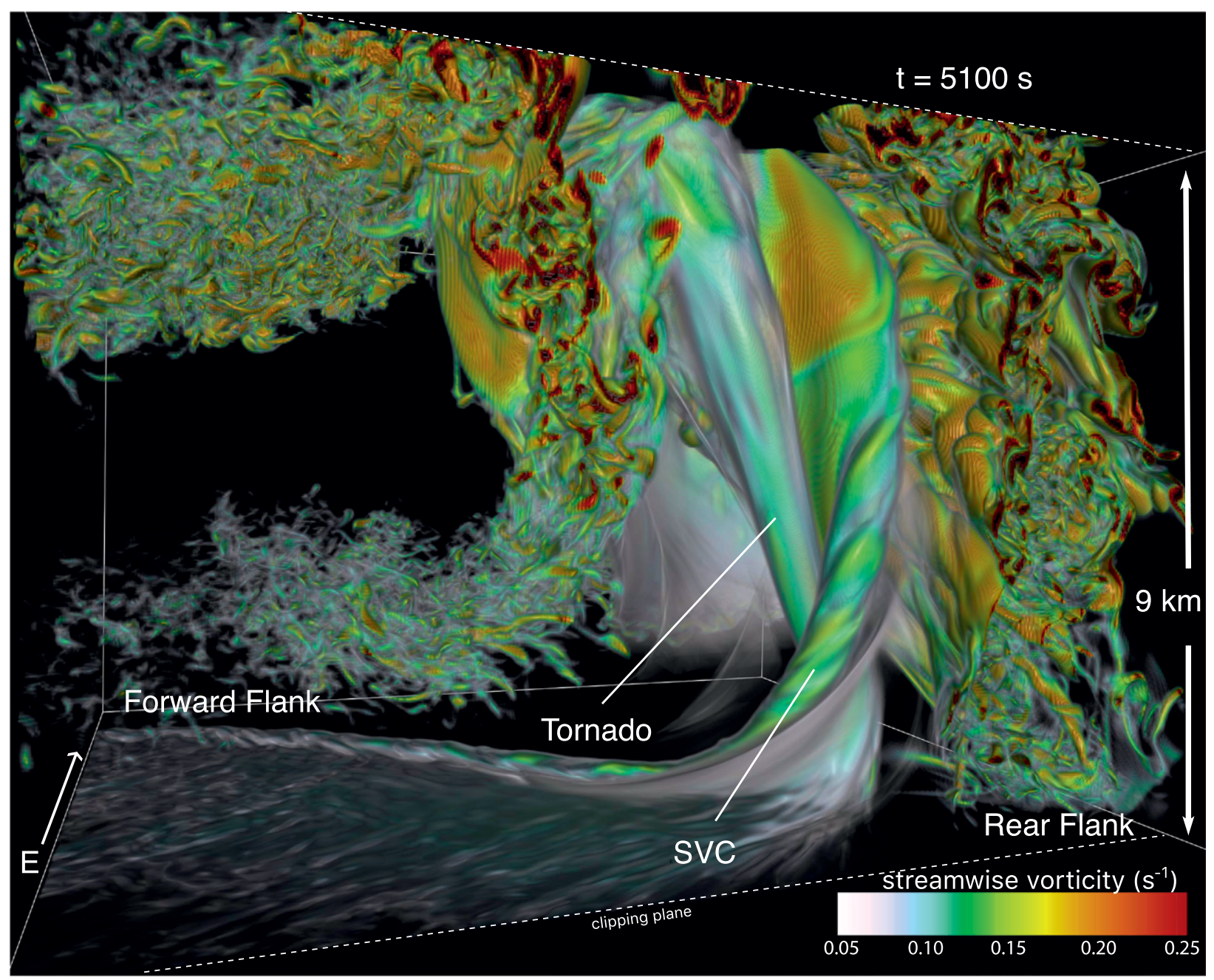

FIG. 10. Volume-rendered storm-relative 3D streamwise vorticity at $t=5,100 \mathrm{~s}$, looking toward the southeast. The tornado and streamwise vorticity current are indicated. The broad region of positive streamwise vorticity just above ground level corresponds to the area of long horizontal vorticity vectors to the north of the tornado in Fig. $6 \mathrm{c}$.

tornadic vortex signatures could be the most common type.

In summary, tornadogenesis occurs in concert with a complex process of cyclonic vertical vorticity accumulation at an inflection point that develops along the FFDB. The timing and location of tornadogenesis also appear associated with the intensification and location of abrupt tilting of the SVC. Additionally, a potentially important factor supporting tornadogenesis and early intensification appeared to involve a rearward-spreading horizontal sheet of low-level streamwise vorticity that originates within the forward-flank outflow. This air converges toward the updraft where it is tilted vertically, becoming part of the tornado's circulation. Contributing processes may not be limited to the mechanisms cited above [e.g., baroclinically generated vorticity that gets tilted into the vertical within a downdraft that converges on the tornado via the Davies-Jones and Brooks (1993) mechanism]. Much more detailed quantitative analysis is underway to examine these processes as well as processes occurring in the RFD as they relate to tornadogenesis and intensification.

Tornado structure and evolution. After genesis, a marked intensification stage commences between $t=4,900$ and $5,130 \mathrm{~s}$, during which time the tornado intensifies to EF4 strength (Fig. 9). By 5,130 s, peak storm-relative surface winds in excess of $80 \mathrm{~m} \mathrm{~s}^{-1}$ bound a circular region of pressure deficit exceeding $60 \mathrm{hPa}$, and vertical vorticity surpasses $2.5 \mathrm{~s}^{-1}$. During this stage, the tornado central pressure decreases at a rate of about

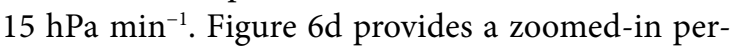
spective of the path and intensification of the young tornado manifest in its pressure deficit history relative to the storm's low-level structure. At $t=5,100 \mathrm{~s}$, 


\section{VISUALIZATION AND ANIMATION}

To simplify postprocessing, visualization, and analysis, middleware was developed to provide easy access to the model output on disk without necessitating any intermediate conversions or requiring a priori knowledge of the structure and format of the raw model data. This facilitated the use of 3D visualization and analysis tools such as Vislt (Childs et al. 2012) and Vapor (Clyne et al. 2007), both of which were used to create many of the figures in this paper.
Both Vislt and Vapor contain the ability to display volume-rendered 3D data. Volume rendering (Drebin et al. 1988; Lichtenbelt et al. 1998) provides capabilities for the exploration of 3D data beyond what can be achieved using isosurfaces, which have been traditionally utilized in the display of 3D fields of convective storms. While computationally expensive, advances in both central processing unit and graphics processing unit technology have made it possible to provide very high-quality, volumerendered imagery from dense 3D data. Volume rendering excels at representing semi-opaque fields that do not behave as solid surfaces such as cloud and rain. Further, by carefully selecting threshold values for color and opacity, both the inner and outer structure of fields such as vorticity magnitude can be displayed concurrently, highlighting regions of small, moderate, and large values of vorticity in a single image. the tornado's condensation funnel has just reached the surface beneath its parent wall cloud, as seen in Fig. 11. The tornado has a single-celled structure and is embedded within an updraft. As during tornadogenesis, cyclonic vorticity regions from the VVS continue moving into the tornado and the tornado remains in a similar location as during genesis with respect to the location of strong SVC tilting within the low-level mesocyclone (Fig. 8f).

Tornado intensification slows markedly between 5,130 and 5,600 s (Fig. 9). During this phase, the tornado continues to display single-cell structure and exhibits storm-relative winds in the vicinity of $80 \mathrm{~m} \mathrm{~s}^{-1}$. The position of the tornado with respect to the SVC remains largely unchanged and the VVS with attendant rearward-moving vortices continues to provide vertical vorticity to the tornado along the storm's FFDB (Fig. 12a).

A much longer tornado intensification period begins around 5,600 s and extends over the next approximate $16 \mathrm{~min}$ (Fig. 9). For much of this phase, storm-relative EF5 winds are indicated. The VVS during this extended intensification period has become very active with a markedly increased accumulation of vertical vorticity along the FFDB. Cyclonic vertical vorticity continues to be absorbed into the tornado as vorticity is extruded from likesigned vortices and vorticity patches moving into it (e.g., Corcos and Sherman 1984; McWilliams 1984; Lee and Wilhelmson 1997). As shown in Fig. 12, the

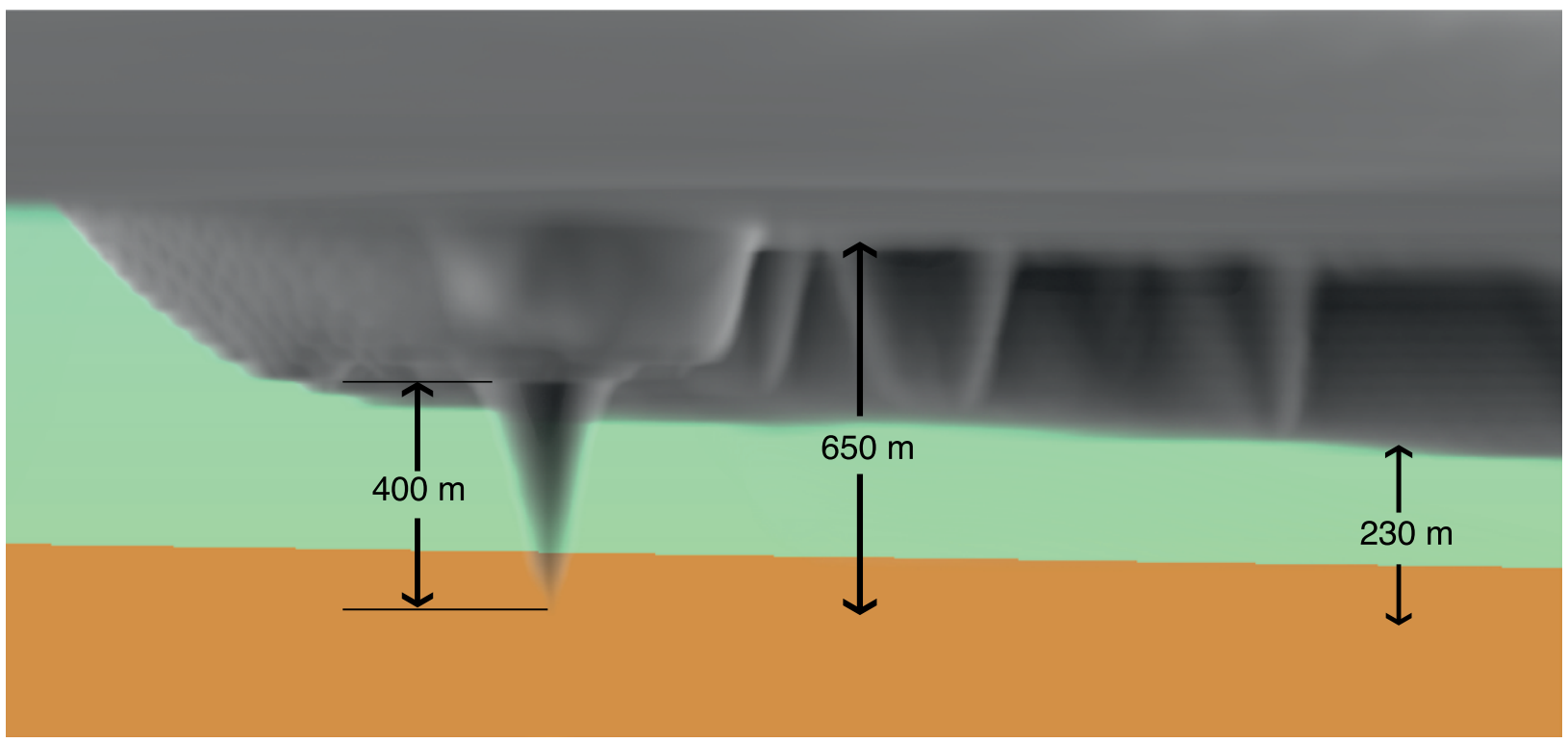

FIG. II. Tornado condensation funnel, as visible in the cloud mixing ratio field, at $t=5,100 \mathrm{~s}$. View is looking toward the northwest. At this time, the visible tornado condensation funnel is $400 \mathrm{~m}$ in length, with the cloud base outside of the wall cloud and tail cloud extending to $650 \mathrm{~m} \mathrm{AGL}$. The base of the tail cloud along the storm's forward flank is 230 m AGL. 
tornado has retained a similar SVC-relative position, but during this phase the tornado assumes a more erect orientation and is in better alignment under and within the strong, deep updraft. Early in this phase $(\sim 5,650 \mathrm{~s})$ the tornado begins a top-down intensification period from a single-cell to a twocelled structure, as evinced by animations of vorticity magnitude and the presence of a circular downdraft in the center of the tornado. During the transition, peak tornadic ground-relative winds at the surface increase steadily, reaching values averaging $100 \mathrm{~m} \mathrm{~s}^{-1}$ from $t=6,000$ to $6,300 \mathrm{~s}$. The tornado maintains EF5 strength for $38 \mathrm{~min}$ and attains a peak instantaneous storm-relative wind speed of $143 \mathrm{~m} \mathrm{~s}^{-1}$ at $6,580 \mathrm{~s}$ (Fig. 12c). This peak wind event, which rises and falls over a 10-s period, is associated with an anticyclonic vortex originating along the FFDB. Like other anticyclonic-cyclonic interactions in this region, the vortex is not absorbed into the tornado's flow, but is swept cyclonically around the outer periphery of the tornado. The vortex is observed to make a full rotation around the tornado before being tilted horizontally and lifted upward. The translational velocity of the anticyclonic vortex relative to the tornado appears responsible for this maximum peak wind, which occurs at the interface between the two vortices.

We explore the structure of the tornado in Fig. 13, which presents a volume-rendered view of the bottom $10 \mathrm{~km}$ of the tornado at the time of maximum storm-relative surface winds. Vortex breakdown is evident above $2 \mathrm{~km}$ AGL, as indicated by the appearance of tightly coupled vortices rotating about the central axis clearly visible between 2 and $4 \mathrm{~km}$ AGL. This is reminiscent of vortex breakdown above
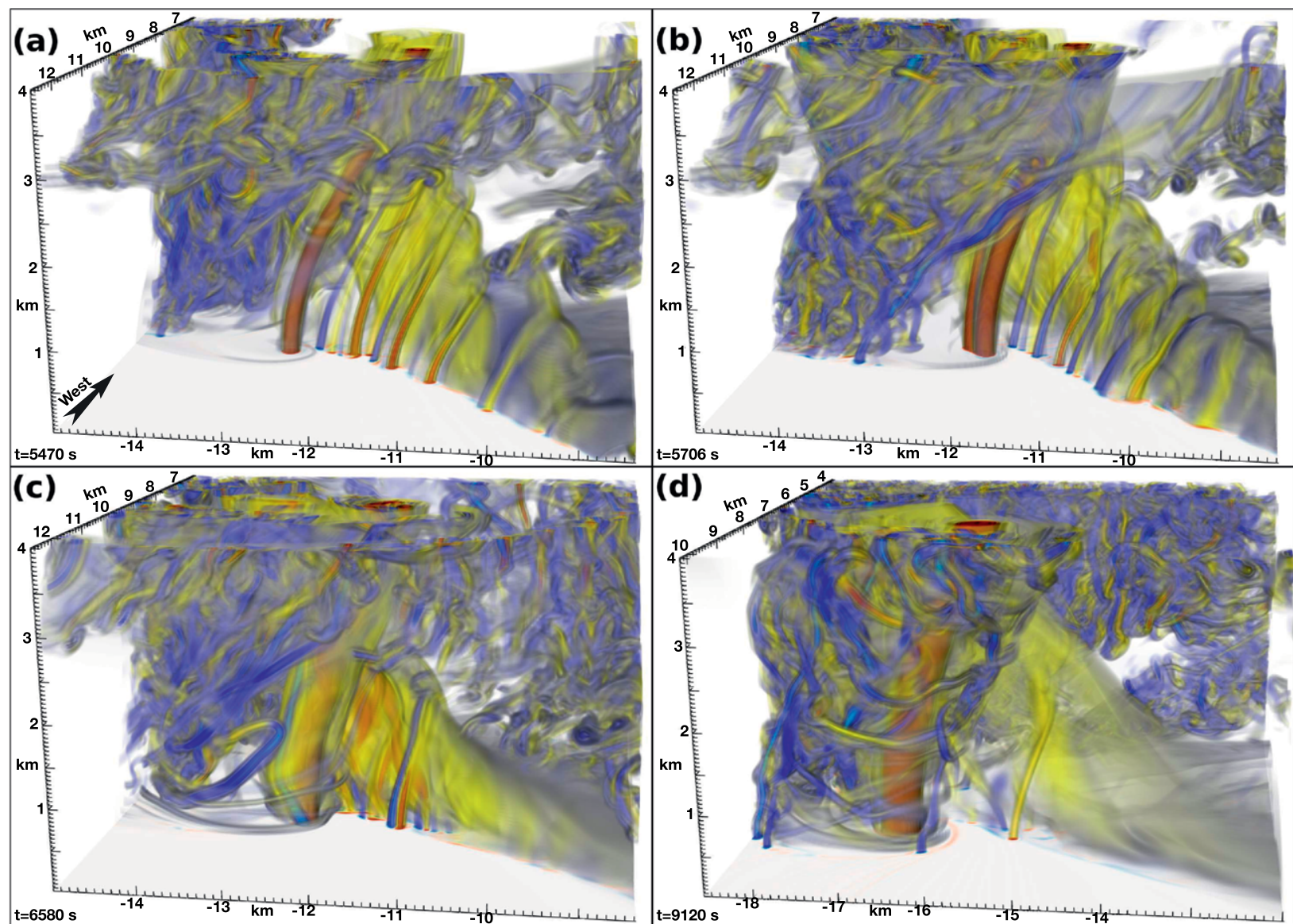

(d)

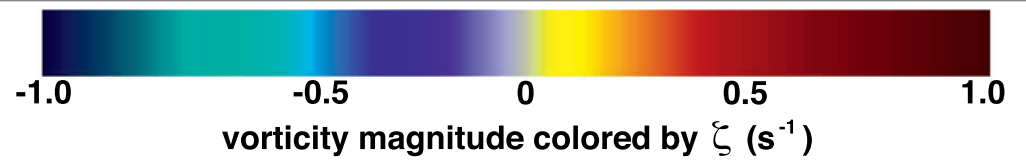

FIG. I2. Volume-rendered vorticity magnitude colored by the vertical component of vorticity (values of vorticity magnitude less than $0.025 \mathrm{~s}^{-1}$ are not shown). (a) $t=5,470 \mathrm{~s}$. (b) $t=5,706 \mathrm{~s}$. (c) $t=6,580 \mathrm{~s}$, at which point storm-relative winds peak at $143 \mathrm{~m} \mathrm{~s}^{-1}$ at the interface between the tornado and an anticylonic vortex, visible near the ground between the viewer and the tornado. (d) $t=9,120 \mathrm{~s}, 42 \mathrm{~min}$ after (c), indicating a configuration that has not varied considerably over time during the mature stage. 
a single-celled vortex as discussed, for example, in Rotunno (2013; see their Fig. 15). But there is also a central axis downdraft extending to the surface, indicating a two-celled vortex [as in Rotunno (2013), their Figs. 9c,d]. The presence of both features together suggests the possibility of nested corner flow vortex structure as described in section 3 of Lewellen and Lewellen (2007), which would allow an inner high-swirl corner flow (with central downdraft to the surface) to be embedded within a largerscale low-swirl corner flow (with vortex breakdown aloft). The experiments described by Rotunno (2013) with free- versus no-slip surface boundary conditions for an idealized Fiedler chamber (Fiedler 1994) indicated that, for simulations using moderate swirl ratios (Church et al. 1979; Snow et al. 1980), the center downdraft in the free-slip simulation (as in the simulation presented herein) extended to ground level, in contrast with comparative no-slip simulations in which the center downdraft is suspended aloft above a region of rapidly ascending air. It is therefore possible that the application of surface friction into future ultra-high-resolution tornadic supercell simulations might result in a different internal tornado structure.

The pressure perturbation field (Fig. 13c) indicates the largest pressure deficit is located near the ground, also consistent with the free-slip simulations described in Rotunno (2013). Pressure deficits associated with the tightly coupled, intertwined cyclonic vortices are evident, with these vortices embedded within a broader area of low pressure associated with the tornado cyclone and the core of the supercell's mesocyclone. A lobe of low pressure extending along and behind the FFDB is also readily apparent in the pressure field. This region of elevated low pressure coincides with the upward branch of the SVC that is shown at $t=7,100 \mathrm{~s}$ in Fig. 14. In this figure, parcels are released every $2 \mathrm{~s}$ in an $x-z$ patch that is oriented roughly normal to the mean storm-relative flow within the SVC immediately behind the FFDB.

Throughout the simulation, regions of concentrated horizontal vorticity originating primarily 


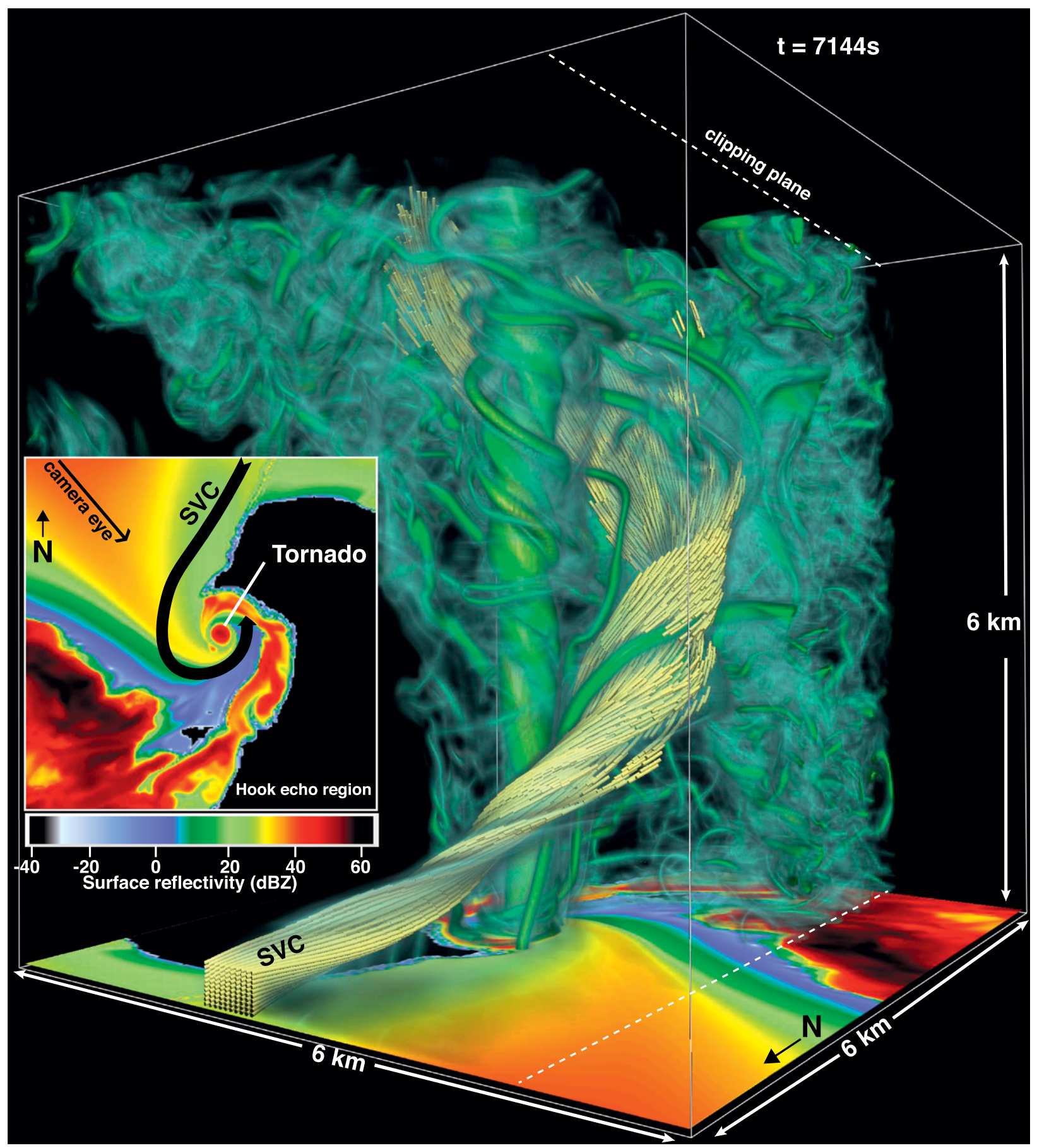

FIG. 14. Volume-rendered vorticity magnitude and trajectory locations at $t=7,144 \mathrm{~s}$ and surface reflectivity (dBZ; color-filled surface). Inset image indicates surface reflectivity and the approximate location of the SVC projected onto the surface.

from the rear flank of the storm along the edge of rear-flank internal surges are occasionally revealed in the cloud mixing ratio field, ostensibly as a result of the cyclostrophic pressure drop in these vortices. Evidence that this process might occur in actual tornadoes is shown in Fig. 15, which compares an observed tornado (Fig. 15a) and the simulated tornado
(Fig. 15b) during a period where a horizontal ring of cloud condensate is observed to ascend around the periphery of the tornado cyclone. This ring of condensate is associated with an intense horizontally oriented vortex ring, indicated by the red arrow in Fig. 15c. The evolutionary time scale of these observed and simulated ascending horizontal vortex rings is 


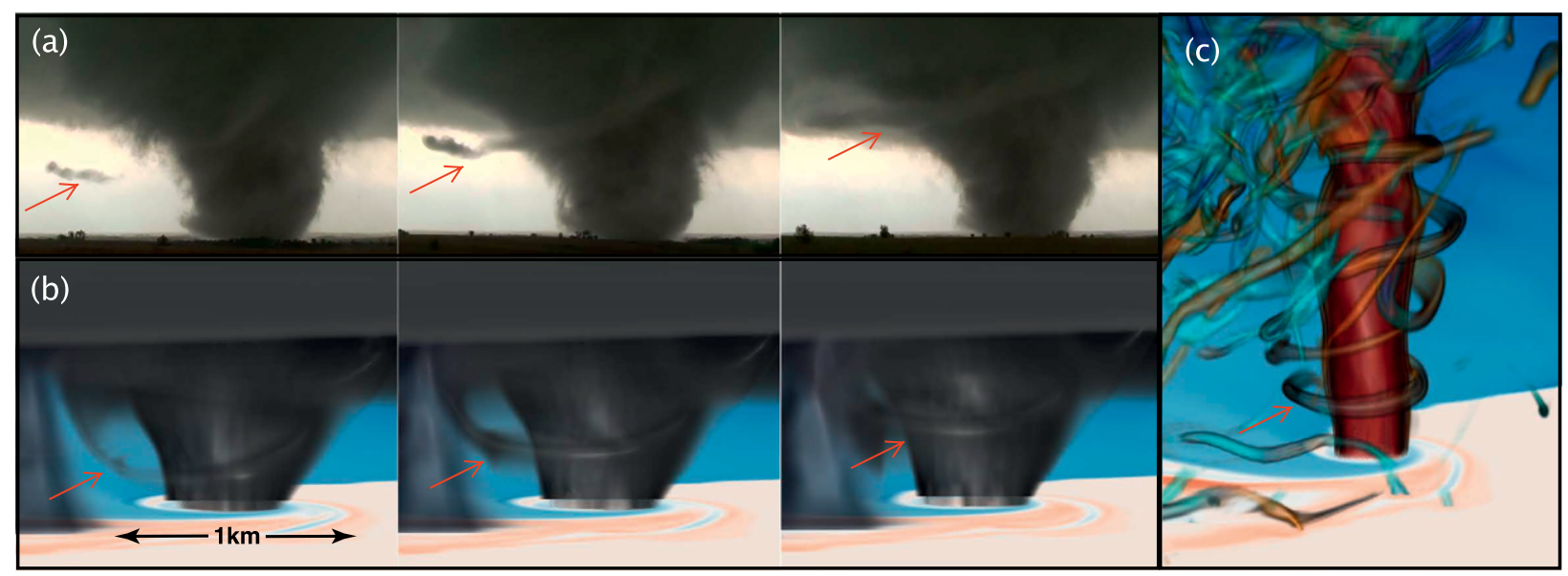

FIG. I5. Comparison between (a) observed ascending horizontal vorticity ring (indicated by red arrow) in the 14 Apr 2012 Langley, Kansas, tornado and (b) similar feature in the simulated cloud field. Video frames are in $\sim 6-s$ intervals, and simulated cloud and rain fields are in 4-s intervals, from $t=7,252 \mathrm{~s}$ to $t=7,260 \mathrm{~s}$. (c) Vorticity magnitude colored by $\zeta$ (values less than 0.25 are not shown) at $t=7,256 \mathrm{~s}$ and surface $\theta^{\prime}$ (color-filled surface). Video captures from video shot by Paul Samaras, used with permission.

roughly similar. Video documentation at close range of this tornado that occurred near Langley, Kansas, on 14 April 2012 showed horizontal vortices wrapping around the tornado periodically during much of its mature phase. While the origin of the horizontal vortex in the video captures cannot be ascertained, the visual similarity between the observed tornado structure and the simulated tornado is quite striking.

Overall storm structure at low levels remains generally consistent during the long maintenance stage of this tornado (Fig. 12d); however, some differences are worth noting. By $t=9,120 \mathrm{~s}$, the vorticity magnitude present in the SVC has decreased, as indicated by the increased transparency of the volume-rendered vorticity field, and fewer cyclonic misocyclonic vortices are present along the FFDB. Further, the tornado has become increasingly surrounded by horizontal and vertical vortices originating from the rear flank that have been cyclonically advected around the periphery of the tornado.

Figure 16 shows the volume-rendered cloud water mixing ratio field and surface potential temperature perturbation $\left(\theta^{\prime}\right)$ field looking down the storm's FFDB toward the end of the maintenance phase. During this 15-min period, the tornado's condensation funnel fluctuates between wide cone and thin rope structures pendant from the wall cloud. The condensation funnel nearly disappears at times and does not extend to ground level for several minutes (the tornado is enshrouded by rain during this period). This change in the tornado condensation funnel corresponds to fluctuations in maximum instantaneous near-surface winds, which vary between 65 and $100 \mathrm{~m} \mathrm{~s}^{-1}$.
Tornado decay. In the minutes leading up to tornado decay, the vortex is generally vertically erect along the tornado's length, with only slight side-to-side "wobbles" as visualized at $t=11,796 \mathrm{~s}$ (Fig. 17a). Storm-relative surface flow indicates a nearly circular flow pattern associated with the tornado. The tornado at this time is enshrouded in rain and is surrounded by the storm's outflow at the surface. Just 84 s later (Fig. 17b), the vortex exhibits kinks just above the surface and aloft, indicating the beginning of a breakdown in its structure. Over the next $70 \mathrm{~s}$ (Figs. 17c,d,e) the tornado undergoes rapid decay that is associated with a strong downdraft that encircles the tornado and is centered at approximately $1 \mathrm{~km}$ AGL (not shown). This downdraft, which coincides with a burst of heavy rain, descends to the ground and a strongly divergent outflow pattern resembling an intense microburst is evident at $t=11,984 \mathrm{~s}$ (Fig. 18). It is noteworthy that this downdraft development and concurrent tornado decay occurs in conjunction with changes in the SVC. A decline in SVC strength, alluded to in the late-mature phase of the tornado, accelerates dramatically in the decay stage, associated with a marked decrease in the $\theta_{\rho}$ gradient in the forward-flank cold pool. The model was run for $500 \mathrm{~s}$ following tornado demise where no further tornadic activity occurred.

SUMMARY AND DISCUSSION. It is now possible to numerically simulate and visualize thunderstorms where genesis, maintenance, and decay of a violent tornado occur within the simulated storm with a reasonable degree of physical similarity to 


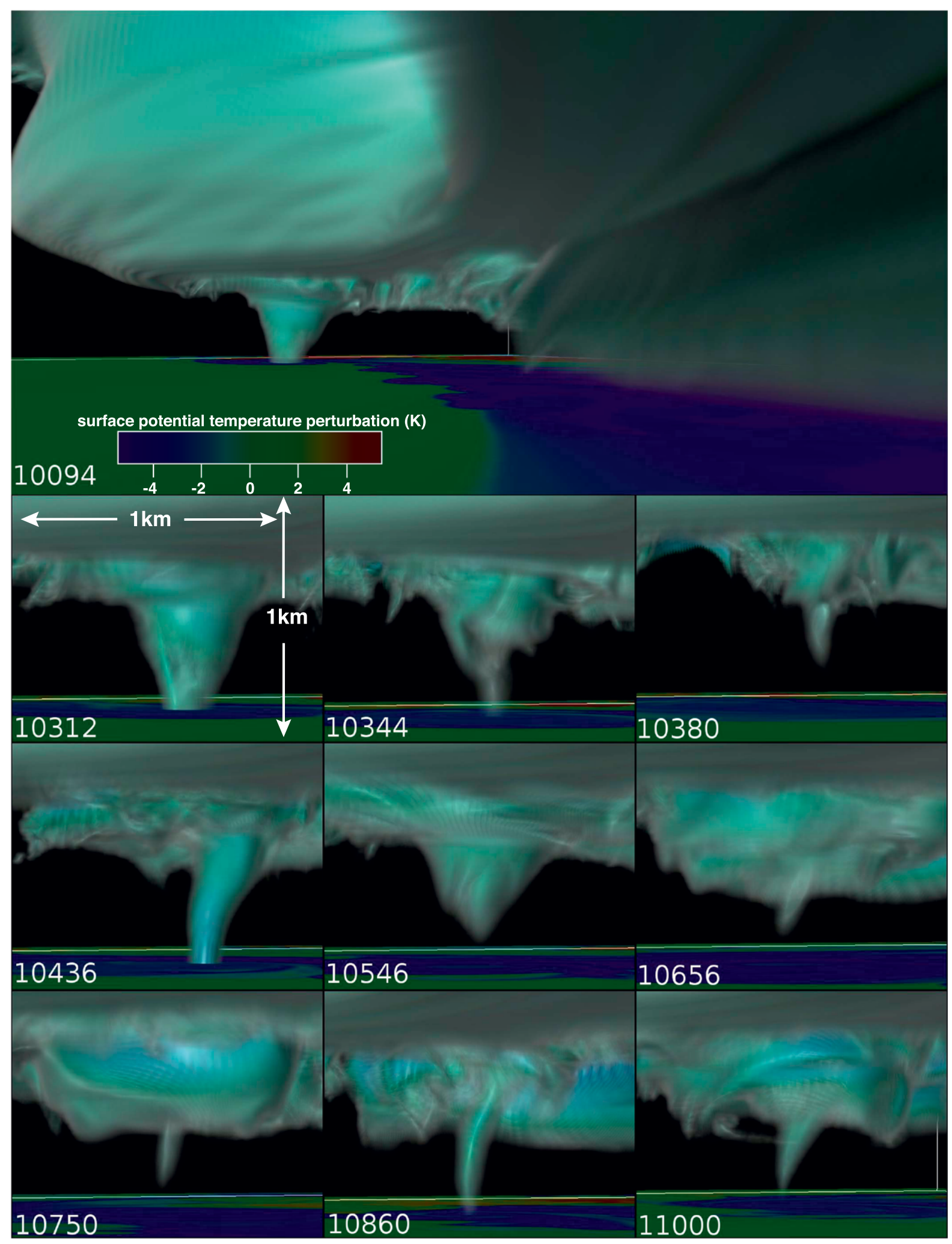

FIG. 16. Volume-rendered cloud mixing ratio and surface potential temperature perturbation from $t=10,094$ to II,000 s. View is toward the southwest, along the forward-flank downdraft boundary (FFDB). 


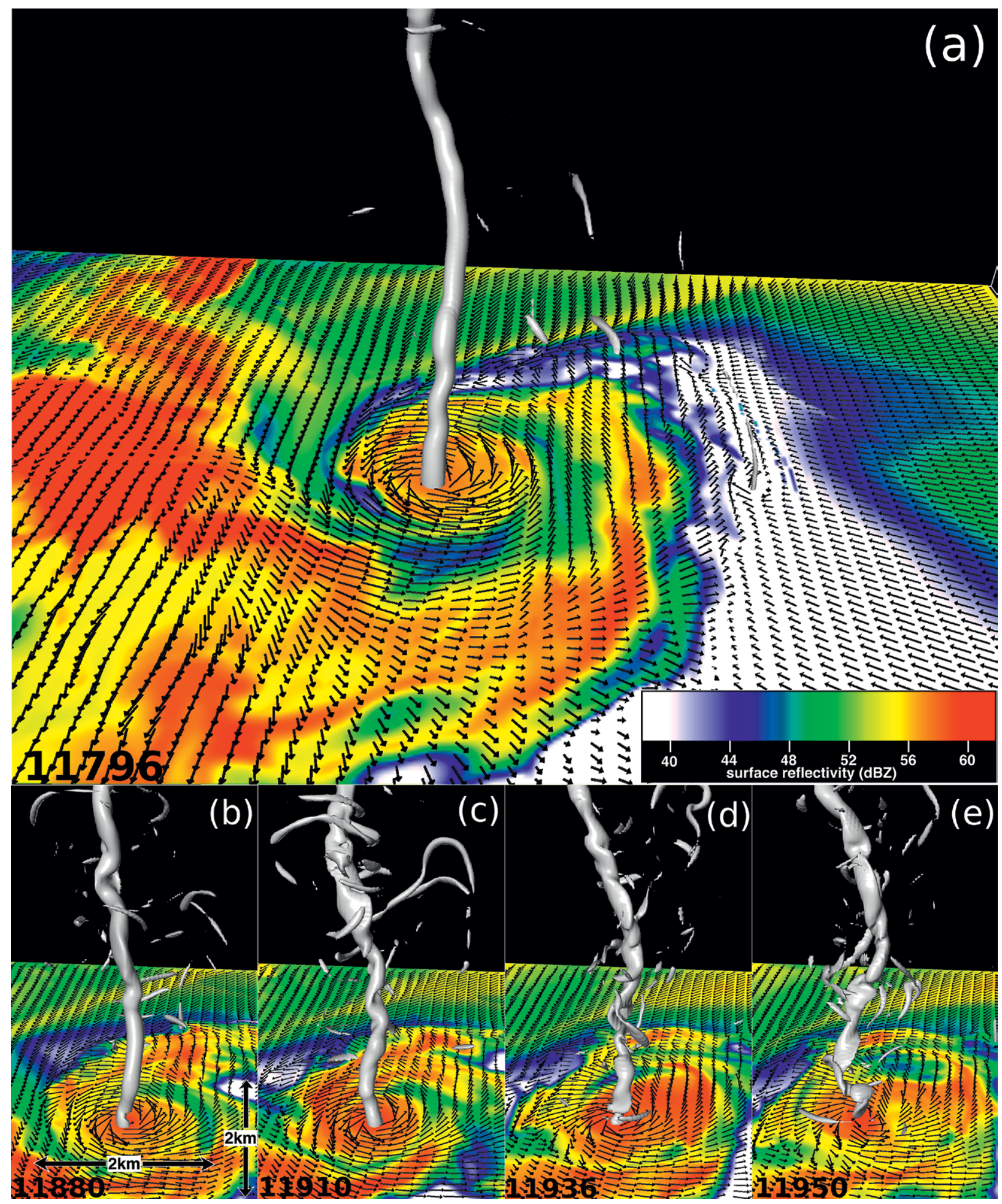

FIG. 17. $1.0 \mathrm{~s}^{-1}$ vorticity magnitude isosurface (white), storm-relative surface wind vectors (drawn approximately every other grid point), and surface reflectivity from 40 to $60 \mathrm{dBZ}$ from $t=11,796$ to $11,950 \mathrm{~s}$. View is looking toward the north. The panels display the bottom $3 \mathrm{~km}$ of the tornado vortex. 
observed storms. A combination of factors made this possible: Blue Waters, a supercomputer with the processing speed and I/O framework to handle cloud modeling simulations utilizing billions of grid points and hundreds of terabytes of data; CM1, a cloud model that efficiently scales to hundreds of thousands of processes and that contains high-order numerics and dual-moment microphysics; a data framework for writing and reading hundreds of terabytes of data built around the HDF5 scientific data format; and VisIt and Vapor, visualization software that are able to handle large datasets and, importantly, produce high-quality volume-rendered imagery. The model was initialized with a sounding extracted from the RUC model, which provided a representative storm environment for a documented violent tornado producing supercell. The simulated storm produced a long-track EF5 tornado, which lasted for approximately $2 \mathrm{~h}$ in the simulation.

During tornadogenesis, there were several salient processes evident in the model fields. The inflection point between the rear- and forward-flank downdraft boundaries was a focal point for the accumulation of vertical vorticity through a process where cyclonic vortices and vorticity patches moving rearward along the FFDB/VVS merged into the incipient tornadic vortex. The inflection point was also a focal region for enhanced near-surface convergence of streamwise vorticity originating within the cold pool. The intensification of the low-level updraft occurred roughly coincident with the development and intensification of a "current" of streamwise vorticity (the SVC) originating along the FFDB that was tilted into the storm's updraft. The location of tornadogenesis occurred very near the SVC upward tilting region.

The maintenance phase of the tornado lasted over $1.5 \mathrm{~h}$ and has been topically described. Many of the features described in the genesis phase of the storm are maintained through much of the maintenance stage, including the flow of cyclonic vertical vorticity along the FFDB that was absorbed into the tornado, the SVC and SVC-relative position of the tornado that became more optimally aligned under and within the strong deep updraft, and the ingestion of streamwise vorticity along and behind the storm's forward-flank downdraft boundary. Tornado decay occurred rapidly as the tornado moved rearward and became surrounded by large amounts of rain within the cold pool and was associated with a strong low-level downdraft that resulted in a divergent, microburst-like outflow. It is noteworthy that this downdraft development and concurrent tornado decay occurred in conjunction with a dramatic weakening in the SVC.
These results provide encouragement that future simulations of other violent tornadic supercell storms with high fidelity are possible. Simulations within the same environment using 20 -m grid spacing that are currently being analyzed have revealed similar long-track, violent tornadoes exhibiting even more realistic-looking structure, including multiple-vortex structures strikingly similar to those observed in the field. However, many challenges remain. To adequately apply surface friction, a much finer vertical mesh will be required for realistic near-ground horizontal winds that experience surface drag over a shallow depth. CM1 does not centrifuge hydrometeors, resulting in a tornado that occasionally becomes filled with rain in its core; modifications are planned to enable this functionality. The robustness of these results in terms of the physical parameterizations chosen (Table 1) is not yet known and will be the subject of future research.

Several physical processes occurring in the FFD/ FFDB were described above, and the relative importance of these processes in tornadogenesis, maintenance, and decay is the focus of additional analysis. Other processes occurring in the RFD/RFDGF (some not presented here) were also seen in the simulation, and their role in the development and evolution of the tornado is the subject of ongoing investigation. Environments beyond that of 24 May 2011 will be explored and compared to observations and the results from this simulation to investigate how commonly the physical processes modeled in this case occur in other real-world and simulated events.

ACKNOWLEDGMENTS. We thank Patrick Skinner, David Lewellen, and an anonymous reviewer, whose comments and suggestions were helpful in improving the paper. We are grateful to Louis Wicker for providing us with the sounding used in this modeling effort and for a number of valued discussions in the early stages of this research. We are especially appreciative to George Bryan for his contributions in providing the source code to CM1 for use by the meteorological research community and for the generous use of his time in answering questions about the model over the last decade. This work benefited from discussions with Rich Rotunno and Morris Weisman of NCAR. David Bock of the National Center for Supercomputing Applications created the image in Fig. 2 utilizing his own volume rendering software on XSEDE hardware. We additionally acknowledge the photography and videography contributions of the 17 June 2014 Carter County Montana tornadic supercell by Roger Hill and Ryan Lueck and the 14 April 2012 Langley, Kansas, videography by Paul Samaras.

We thank all of the programmers who have provided the following open-source software free of charge that were 


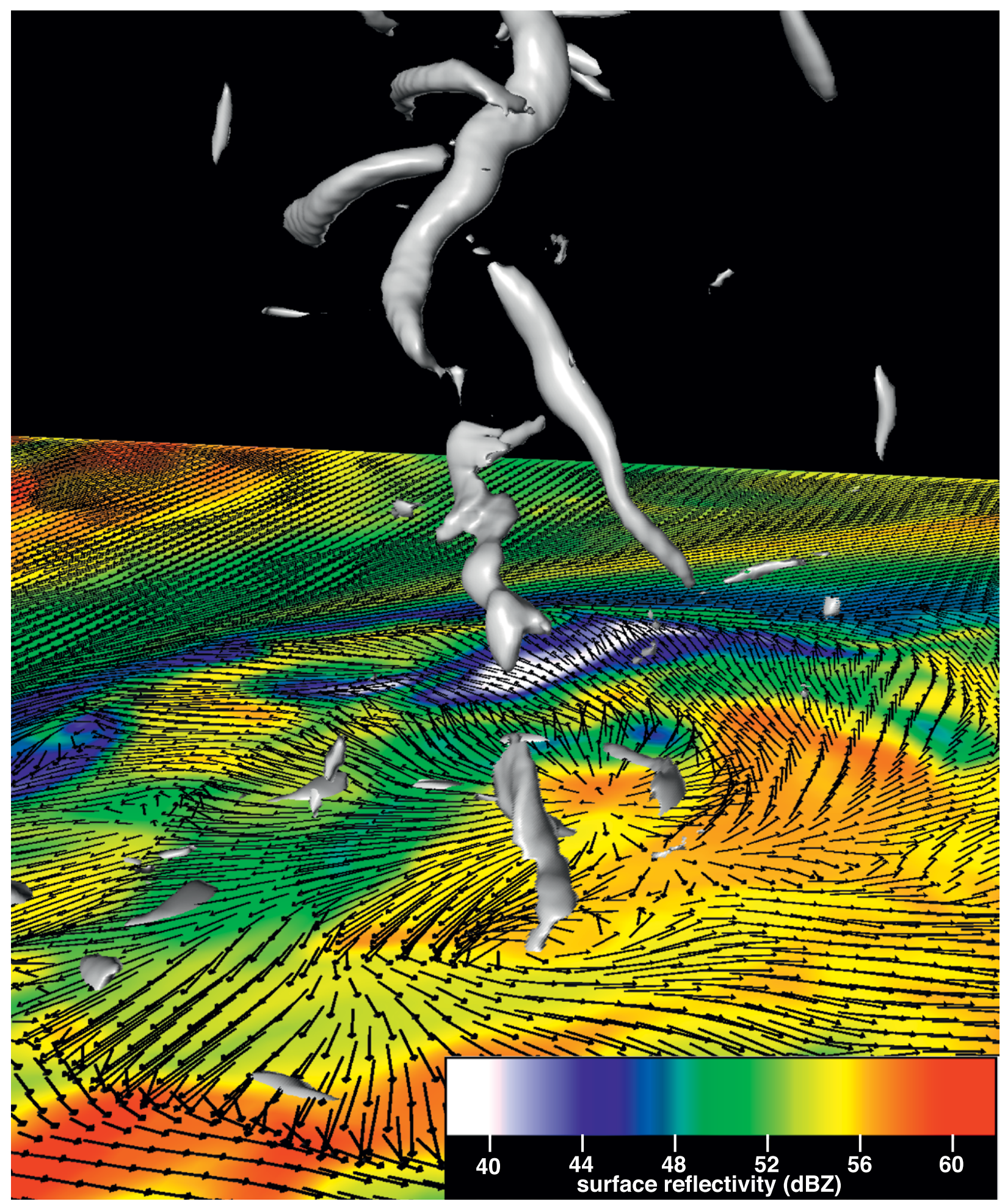

FıG. 18. As in Fig. I7, but at $t=11,984 \mathrm{~s}$.

indispensable for this work: Linux, FFmpeg, ImageMagick, Vapor, VisIt, NCAR Graphics, matplotlib, Python, SHARPpy, and GIMP. This research was supported by NSF Grants ATM-0843566, OCI-0725070, and ACI-1238993. This research is part of the Blue Waters sustained-petascale computing project, which is supported by the National Science Foundation and the state of Illinois. Blue Waters is a joint effort of the University of Illinois at UrbanaChampaign and its National Center for Supercomputing Applications. 


\section{REFERENCES}

Batchelor, G. K., 1967: An Introduction to Fluid Dynamics. Cambridge University Press, 615 pp.

Beck, J., and C. Weiss, 2013: An assessment of lowlevel baroclinity and vorticity within a simulated supercell. Mon. Wea. Rev., 141, 649-669, doi:10.1175 /MWR-D-11-00115.1.

Benjamin, S. G., and Coauthors, 2004: An hourly assimilation-forecast cycle: The RUC. Mon. Wea. Rev., 132, 495-518, doi:10.1175/1520-0493(2004)132<0495:AH ACTR $>2.0 . C O ; 2$.

Bluestein, H. B., C. C. Weiss, and A. L. Pazmany, 2003: Mobile Doppler radar observations of a tornado in a supercell near Bassett, Nebraska, on 5 June 1999. Part I: Tornadogenesis. Mon. Wea. Rev., 131, 2954-2967, doi:10.1175/1520-0493(2003)131<2954:MDROOA $>2.0 . \mathrm{CO} ; 2$.

Bode, B., M. Butler, T. Dunning, T. Hoefler, W. Kramer, W. Gropp, and W.-M. Hwu, 2013: The Blue Waters super-system for super-science. Contemporary High Performance Computing: From Petascale toward Exascale, J. S. Vetter, Ed., Chapman and Hall/CRC, 339-366.

Brandes, E. A., 1977: Gust front evolution and tornado genesis as viewed by Doppler radar. J. Appl. Meteor., 16, 333-338, doi:10.1175/1520-0450(1977)016<0333:GF EATG>2.0.CO;2.

— 1978: Mesocyclone evolution and tornadogenesis: Some observations. Mon. Wea. Rev., 106, 995-1011, doi:10.1175/1520-0493(1978)106<0995:MEATSO $>2.0 . \mathrm{CO} ; 2$

Bryan, G. H., 2008: CM1 homepage. Accessed 17 July 2015. [Available online at www2.mmm.ucar.edu /people/bryan/cm1/.]

—, and J. M. Fritsch, 2002: A benchmark simulation for moist nonhydrostatic numerical models. Mon. Wea. Rev., 130, 2917-2928, doi:10.1175/1520 -0493(2002)130<2917:ABSFMN>2.0.CO;2.

Childs, H., and Coauthors, 2012: VisIt: An end-user tool for visualizing and analyzing very large data. High Performance Visualization: Enabling Extreme-Scale Scientific Insight, E. W. Bethel, H. Childs, and C. Hansen, Eds., Chapman and Hall/CRC, 357-372.

Church, C. R., J. T. Snow, G. L. Baker, and E. M. Agee, 1979: Characteristics of tornado-like vortices as a function of swirl ratio: A laboratory investigation. J. Atmos. Sci., 36, 1755-1776, doi:10.1175/1520 -0469(1979)036<1755:COTLVA>2.0.CO;2.

Clyne, J., P. Mininni, A. Norton, and M. Rast, 2007: Interactive desktop analysis of high resolution simulations: Application to turbulent plume dynamics and current sheet formation. New J. Phys., 9, 301, doi:10.1088/1367-2630/9/8/301.
Corcos, G. M., and F. S. Sherman, 1984: The mixing layer: Deterministic models of a turbulent flow. Part 1. Introduction and the two-dimensional flow. J. Fluid Mech., 139, 29-65, doi:10.1017/S0022112084000252.

Dahl, J. M. L., M. D. Parker, and L. J. Wicker, 2014: Imported and storm-generated near-ground vertical vorticity in a simulated supercell. J. Atmos. Sci., 71, 3027-3051, doi:10.1175/JAS-D-13-0123.1.

Davies-Jones, R., and H. Brooks, 1993: Mesocyclogenesis from a theoretical perspective. The Tornado: Its Structure, Dynamics, Prediction, and Hazards, Geophys. Monogr., Vol. 79, Amer. Geophys. Union, 105-114, doi:10.1029/GM079p0105.

Dowell, D. C., and H. B. Bluestein, 2002: The 8 June 1995 McLean, Texas, storm. Part II: Cyclic tornado formation, maintenance, and dissipation. Mon. Wea. Rev., 130, 2649-2670, doi:10.1175/1520 -0493(2002)130<2649:TJMTSP>2.0.CO;2.

- , Y. P. Richardson, and J. M. Wurman, 2002 Observations of the formation of low-level rotation: The 5 June 2001 Sumner County, Kansas tornado. 21st Conf. on Severe Local Storms, San Antonio, TX, Amer. Meteor. Soc., 12.3. [Available online at https:// ams.confex.com/ams/SLS_WAF_NWP/techprogram /paper_47335.htm.]

Drebin, R. A., L. Carpenter, and P. Hanrahan, 1988: Transfer functions on a logarithmic scale for volume rendering. Proc. 15th Annual Conf. on Computer Graphics and Interactive Techniques, New York, NY, ACM, 65-74, doi:10.1145/54852.378484.

Emanuel, K. A., 1994: Atmospheric Convection. 1st ed. Oxford University Press, $592 \mathrm{pp}$.

Fiedler, B. H., 1994: The thermodynamic speed limit and its violation in axisymmetric numerical simulations of tornadolike vortices. Atmos.-Ocean, 32, 335-359, doi:10.1080/07055900.1994.9649501.

Finley, C. A., and B. D. Lee, 2004: High resolution mobile mesonet observation of RFD surges in the June 9 Basset, Nebraska supercell during Project ANSWERS 2003. 22nd Conf. on Severe Local Storms, Hyannis, MA, Amer. Meteor. Soc., P11.3. [Available online at https://ams.confex.com/ams/11aram22sls /techprogram/paper_82005.htm.]

— W. W. Cotton, and A. Pielke, 2002: Tornadogenesis in a simulated HP supercell. 21st Conf. on Severe Local Storms, San Antonio, TX, Amer. Meteor. Soc., P10.1. [Available online at https://ams.confex.com/ams /SLS_WAF_NWP/techprogram/paper_47216.htm.]

French, M. M., H. B. Bluestein, I. PopStefanija, C. A. Baldi, and R. T. Bluth, 2013: Reexamining the vertical development of tornadic vortex signatures in supercells. Mon. Wea. Rev., 141, 4576-4601, doi:10.1175 /MWR-D-12-00315.1. 
- P. S. Skinner, L. J. Wicker, and H. B. Bluestein, 2015: Documenting a rare tornado merger observed in the 24 May 2011 El Reno-Piedmont, Oklahoma, supercell. Mon. Wea. Rev., 143, 3025-3043, doi:10.1175/MWR -D-14-00349.1.

Gaudet, B. J., W. R. Cotton, and M. T. Montgomery, 2006: Low-level mesocyclonic concentration by nonaxisymmetric transport. Part II: Vorticity dynamics. J. Atmos. Sci., 63, 1134-1150, doi:10.1175 /JAS3579.1.

Grzych, M. L., B. D. Lee, and C. A. Finley, 2007: Thermodynamic analysis of supercell rear-flank downdrafts from project ANSWERS. Mon. Wea. Rev., 135, 240-246, doi:10.1175/MWR3288.1.

Hart, J. A., and W. Korotky, 1991: The SHARP workstation vl.50 user's guide. NOAA/National Weather Service Tech. Rep., 30 pp.

HDF Group, 2016: Hierarchical Data Format, version 5. Accessed 29 February 2016. [Available online at www.hdfgroup.org/HDF5/.]

Houser, J. L., H. B. Bluestein, and J. C. Snyder, 2015: Rapid-scan, polarimetric, Doppler radar observations of tornadogenesis and tornado dissipation in a tornadic supercell: The "El Reno, Oklahoma" storm of 24 May 2011. Mon. Wea. Rev., 143, 2685-2710, doi:10.1175/MWR-D-14-00253.1.

Houston, A. L., R. L. Thompson, and R. Edwards, 2008: The optimal bulk wind differential depth and the utility of the upper-tropospheric storm-relative flow for forecasting supercells. Wea. Forecasting, 23, 825-837, doi:10.1175/2008WAF2007007.1.

Klemp, J. B., and R. B. Wilhelmson, 1978a: The simulation of three-dimensional convective storm dynamics. J. Atmos. Sci., 35, 1070-1096, doi:10.1175/1520 -0469(1978)035<1070:TSOTDC>2.0.CO;2.

- , and — 1978b: Simulations of right- and leftmoving storms produced through storm splitting. J. Atmos. Sci., 35, 1097-1110, doi:10.1175/1520 -0469(1978)035<1097:SORALM>2.0.CO;2.

—_ _ , and P. S. Ray, 1981: Observed and numerically simulated structure of a mature supercell thunderstorm. J. Atmos. Sci., 38, 1558-1580, doi:10.1175/1520-0469(1981)038<1558:OANSSO $>2$ .0.CO;2.

Kosiba, K., J. Wurman, Y. Richardson, P. Markowski, P. Robinson, and J. Marquis, 2013: Genesis of the Goshen County, Wyoming, tornado on 5 June 2009 during VORTEX2. Mon. Wea. Rev., 141, 1157-1181, doi:10.1175/MWR-D-12-00056.1.

Kramer, W., M. Butler, G. Bauer, K. Chadalavada, and C. Mendes, 2014: National Center for Supercomputing Applications. High Performance Parallel I/O, Q. K. Prabhat, Ed., Chapman and Hall/CRC, 17-31.
Lee, B. D., and R. B. Wilhelmson, 1997: The numerical simulation of nonsupercell tornadogenesis. Part II: Evolution of a family of tornadoes along a weak outflow boundary. J. Atmos. Sci., 54, 2387-2415, doi:10.1175/1520-0469(1997)054<2387:TNSONT $>2.0 . \mathrm{CO} ; 2$.

—, C. A. Finley, and P. Skinner, 2004: Thermodynamic and kinematic analysis of multiple RFD surges for the 24 June 2003 Manchester, SD cyclic tornadic supercell during Project ANSWERS 2003. 22nd Conf. on Severe Local Storms, Hyannis, MA, Amer. Meteor. Soc., P11.2. [Available online at https://ams .confex.com/ams/11aram22sls/techprogram/paper 82000.htm.]

— - , and T. M. Samaras, 2011: Surface analysis near and within the Tipton, Kansas, Tornado on 29 May 2008. Mon. Wea. Rev., 139, 370-386, doi:10.1175/2010MWR3454.1.

— - _ , and C. D. Karstens, 2012: The Bowdle, South Dakota, cyclic tornadic supercell of 22 May 2010: Surface analysis of rear-flank downdraft evolution and multiple internal surges. Mon. Wea. Rev., 140, 3419-3441, doi:10.1175/MWR-D-11-00351.1.

Lemon, L. R., and C. A. Doswell, 1979: Severe thunderstorm evolution and mesocyclone structure as related to tornadogenesis. Mon. Wea. Rev., 107, 1184-1197, doi:10.1175/1520-0493(1979)107<1184:STEAMS $>2$ .0.CO;2.

Lewellen, D. C., and W. S. Lewellen, 2007: Near-surface intensification of tornado vortices. J. Atmos. Sci., 64, 2176-2194, doi:10.1175/JAS3965.1.

— cal swirl ratio on tornado intensification near the surface. J. Atmos. Sci., 57, 527-544, doi:10.1175/1520 -0469(2000)057<0527:TIOALS>2.0.CO;2.

Lewellen, W. S., D. C. Lewellen, and R. I. Sykes, 1997: Large-eddy simulation of a tornado's interaction with the surface. J. Atmos. Sci., 54, 581-605, doi:10.1175/1520-0469(1997)054<0581:LESOAT $>2.0 . \mathrm{CO} ; 2$

Lichtenbelt, B., R. Crane, and S. Naqvi, 1998: Introduction to Volume Rendering. Prentice-Hall, 236 pp.

Markowski, P. M., J. M. Straka, and E. N. Rasmussen, 2002: Direct surface thermodynamic observations within the rear-flank downdrafts of nontornadic and tornadic supercells. Mon. Wea. Rev., 130, 1692-1721, doi:10.1175/1520-0493(2002)130<1692:DSTOWT $>2.0 . \mathrm{CO} ; 2$.

- and Coauthors, 2012a: The pretornadic phase of the Goshen County, Wyoming, supercell of 5 June 2009 intercepted by VORTEX2. Part I: Evolution of kinematic and surface thermodynamic fields. Mon. Wea. Rev., 140, 2887-2915, doi:10.1175/MWR-D-11-00336.1. 
- and Coauthors, 2012b: The pretornadic phase of the Goshen County, Wyoming, supercell of 5 June 2009 intercepted by VORTEX2. Part II: Intensification of low-level rotation. Mon. Wea. Rev., 140, 2916-2938, doi:10.1175/MWR-D-11-00337.1.

—, Y. Richardson, and G. Bryan, 2014: The origins of vortex sheets in a simulated supercell thunderstorm. Mon. Wea. Rev., 142, 3944-3954, doi:10.1175/MWR -D-14-00162.1.

Marquis, J., Y. Richardson, P. Markowski, D. Dowell, and J. Wurman, 2012: Tornado maintenance investigated with high-resolution dual-Doppler and EnKF analysis. Mon. Wea. Rev., 140, 3-27, doi:10.1175 /MWR-D-11-00025.1.

McWilliams, J. C., 1984: The emergence of isolated coherent vortices in turbulent flow. J. Fluid Mech., 146, 21-43, doi:10.1017/S0022112084001750.

Miles, J. W., and L. N. Howard, 1964: Note on a heterogeneous shear flow. J. Fluid Mech., 20, 331-336, doi:10.1017/S0022112064001252.

Moller, A. R., C. A. Doswell, M. P. Foster, and G. R. Woodall, 1994: The operational recognition of supercell thunderstorm environments and storm structures. Wea. Forecasting, 9, 327-347, doi:10.1175/1520 -0434(1994)009<0327:TOROST>2.0.CO;2.

Moore, G. E., 1965: Cramming more components onto integrated circuits. Electronics, 38, 114-117.

Morrison, H., G. Thompson, and V. Tatarskii, 2009: Impact of cloud microphysics on the development of trailing stratiform precipitation in a simulated squall line: Comparison of one- and two-moment schemes. Mon. Wea. Rev., 137, 991-1007, doi:10.1175/2008MWR2556.1.

National Weather Service, 2011a: Radar images related to the May 24, 2011 tornado outbreak. Accessed 29 October 2015. [Available online at www.srh.noaa .gov/oun/?n=events-20110524-radar.]

— , 2011b: The tornado outbreak of May 24, 2011. Accessed 29 October 2015. [Available online at www .srh.noaa.gov/oun/?n=events-20110524.]

Naylor, J., and M. S. Gilmore, 2012: Convective initiation in an idealized cloud model using an updraft nudging technique. Mon. Wea. Rev., 140, 3699-3705, doi:10.1175/MWR-D-12-00163.1.

Orf, L., R. Wilhelmson, and L. Wicker, 2016: Visualization of a simulated long-track EF5 tornado embedded within a supercell thunderstorm. Parallel Comput., 55, 28-34, doi:10.1016/j.parco.2015.10.014.

Rasmussen, E. N., 2003: Refined supercell and tornado forecast parameters. Wea. Forecasting, 18, 530-535, doi:10.1175/1520-0434(2003)18<530:RSATFP $>2$ $.0 . \mathrm{CO} ; 2$.

— ogy of sounding-derived supercell and tornado forecast parameters. Wea. Forecasting, 13, 1148-1164, doi:10.1175/1520-0434(1998)013<1148:ABCOSD >2 $.0 . \mathrm{CO} ; 2$.

Rotunno, R., 2013: The fluid dynamics of tornadoes. Annu. Rev. Fluid Mech., 45, 59-84, doi:10.1146 /annurev-fluid-011212-140639.

— , and J. B. Klemp, 1982: The influence of the shearinduced pressure gradient on thunderstorm motion. Mon. Wea. Rev., 110, 136-151, doi:10.1175/1520 -0493(1982)110<0136:TIOTSI>2.0.CO;2.

- , and — 1985: On the rotation and propagation of simulated supercell thunderstorms. J. Atmos. Sci., 42, 271-292, doi:10.1175/1520-0469(1985)042<0271:OT $\mathrm{RAPO}>2.0 . \mathrm{CO} ; 2$.

Schenkman, A. D., M. Xue, and A. Shapiro, 2012: Tornadogenesis in a simulated mesovortex within a mesoscale convective system. J. Atmos. Sci., 69, 3372-3390, doi:10.1175/JAS-D-12-038.1.

,$- \ldots$, and M. Hu, 2014: Tornadogenesis in a highresolution simulation of the 8 May 2003 Oklahoma City supercell. J. Atmos. Sci., 71, 130-154, doi:10.1175 /JAS-D-13-073.1.

$\longrightarrow,-$, and D. T. Dawson II, 2016: The cause of internal outflow surges in a high-resolution simulation of the 8 May 2003 Oklahoma City tornadic supercell. J. Atmos. Sci., 73, 353-370, doi:10.1175 /JAS-D-15-0112.1.

Schlesinger, R. E., 1980: A three-dimensional numerical model of an isolated thunderstorm. Part II: Dynamics of updraft splitting and mesovortex couplet evolution. J. Atmos. Sci., 37, 395-420, doi:10.1175/1520 -0469(1980)037<0395:ATDNMO>2.0.CO;2.

Skinner, P. S., C. C. Weiss, M. M. French, H. B. Bluestein, P. M. Markowski, and Y. P. Richardson, 2014: VORTEX2 observations of a low-level mesocyclone with multiple internal rear-flank downdraft momentum surges in the 18 May 2010 Dumas, Texas, supercell. Mon. Wea. Rev., 142, 2935-2960, doi:10.1175/MWR -D-13-00240.1.

,,-- L. J. Wicker, C. K. Potvin, and D. C. Dowell, 2015: Forcing mechanisms for an internal rearflank downdraft momentum surge in the 18 May 2010 Dumas, Texas, supercell. Mon. Wea. Rev., 143, 4305-4330, doi:10.1175/MWR-D-15-0164.1.

Smagorinsky, J., 1963: General circulation experiments with the primitive equations. Mon. Wea. Rev., 91, 99-164, doi:10.1175/1520-0493(1963)091<0099:GC EWTP>2.3.CO;2.

Snow, J. T., C. R. Church, and B. J. Barnhart, 1980: An investigation of the surface pressure fields beneath simulated tornado cyclones. J. Atmos. Sci., 37, 1013-1026, doi:10.1175/1520-0469(1980)037<1013:AI $\mathrm{OTSP}>2.0 . \mathrm{CO} ; 2$. 
Snyder, J. C., H. B. Bluestein, V. Venkatesh, and S. J. Frasier, 2013: Observations of polarimetric signatures in supercells by an X-band mobile Doppler radar. Mon. Wea. Rev., 141, 3-29, doi:10.1175/MWR -D-12-00068.1.

Tanamachi, R. L., P. L. Heinselman, and L. J. Wicker, 2015: Impacts of a storm merger on the 24 May 2011 El Reno, Oklahoma, tornadic supercell. Wea. Forecasting, 30, 501-524, doi:10.1175/WAF-D-14-00164.1.

Thompson, R. L., R. Edwards, J. A. Hart, K. L. Elmore, and P. Markowski, 2003: Close proximity soundings within supercell environments obtained from the Rapid Update Cycle. Wea. Forecasting, 18, 1243 1261, doi:10.1175/1520-0434(2003)018<1243:CPSW SE>2.0.CO;2.

Trapp, R. J., and B. H. Fiedler, 1995: Tornado-like vortexgenesis in a simplified numerical model. J. Atmos. Sci., 52, 3757-3778, doi:10.1175/1520-0469(1995)052 $<3757$ :TLVIAS $>2.0 . \mathrm{CO} ; 2$.

— and without a dynamic pipe effect. J. Atmos. Sci., 54, 113-133, doi:10.1175/1520-0469(1997)054<0113:TW AWAD>2.0.CO;2.

—, E. D. Mitchell, G. A. Tipton, D. W. Effertz, A. I. Watson, D. L. Andra, and M. A. Magsig, 1999: Descending and nondescending tornadic vortex signatures detected by WSR-88Ds. Wea. Forecasting, 14, 625-639, doi:10.1175/1520-0434(1999)014<0625:DA NTVS $>2.0 . \mathrm{CO} ; 2$.

Ward, N. B., 1972: The exploration of certain features of tornado dynamics using a laboratory model. J. Atmos. Sci., 29, 1194-1204, doi:10.1175/1520-0469(1972)029 $<1194$ :TEOCFO>2.0.CO;2.

Weisman, M. L., and J. B. Klemp, 1982: The dependence of numerically simulated convective storms on vertical wind shear and buoyancy. Mon. Wea. Rev., 110, 504-520, doi:10.1175/1520-0493(1982)110<0504:TD ONSC $>2.0 . \mathrm{CO} ; 2$.

- and — 1984: The structure and classification of numerically simulated convective storms in directionally varying wind shears. Mon. Wea. Rev., 112, 2479-2498, doi:10.1175/1520-0493(1984)112<2479:TS $\mathrm{ACON}>2.0 . \mathrm{CO} ; 2$.
Wicker, L. J., and W. C. Skamarock, 1998: A time-splitting scheme for the elastic equations incorporating secondorder Runge-Kutta time differencing. Mon. Wea. Rev., 126, 1992-1999, doi:10.1175/1520-0493(1998)126 $<$ 1992:ATSSFT>2.0.CO;2.

Wilhelmson, R. B., and J. B. Klemp, 1978: A numerical study of storm splitting that leads to long-lived storms. J. Atmos. Sci., 35, 1974-1986, doi:10.1175/1520-0469(1978)035<1974:ANSOSS >2 $.0 . \mathrm{CO} ; 2$.

— severe local storms. Severe Convective Storms, Meteor. Monogr., No. 50, Amer. Meteor. Soc., 123-166.

Wurman, J., and K. Kosiba, 2013: Finescale radar observations of tornado and mesocyclone structures. Wea. Forecasting, 28, 1157-1174, doi:10.1175/WAF -D-12-00127.1.

—, D. Dowell, Y. Richardson, P. Markowski, E. Rasmussen, D. Burgess, L. Wicker, and H. B. Bluestein, 2012: The second verification of the origins of rotation in tornadoes experiment: VORTEX2. Bull. Amer. Meteor. Soc., 93, 1147-1170, doi:10.1175 /BAMS-D-11-00010.1.

— K. Kosiba, P. Robinson, and T. Marshall, 2014: The role of multiple-vortex tornado structure in causing storm researcher fatalities. Bull. Amer. Meteor. Soc., 95, 31-45, doi:10.1175/BAMS-D-13-00221.1.

Xue, M., 2004: Tornadogenesis within a simulated supercell storm. 22nd Conf. Severe Local Storms, Hyannis, MA, Amer. Meteor. Soc., 9.6. [Available online at https://ams.confex.com/ams/11aram22sls /techprogram/paper_81574.htm.]

— , D. Wang, J. Gao, K. Brewster, and K. K. Droegemeier, 2003: The Advanced Regional Prediction System (ARPS), storm-scale numerical weather prediction and data assimilation. Meteor. Atmos. Phys., 82 (1-4), 139-170, doi:10.1007/s00703-001-0595-6.

- M. Hu, and A. D. Schenkman, 2014: Numerical prediction of the 8 May 2003 Oklahoma City tornadic supercell and embedded tornado using ARPS with the assimilation of WSR-88D data. Wea. Forecasting, 29, 39-62, doi:10.1175/WAF-D-13-00029.1. 